TITLE:

\title{
Finite element formulation and analysis for an arterial wall with residual and active stresses
}

$\operatorname{AUTHOR}(\mathrm{S})$ :

Kida, Naoki; Adachi, Taiji

\section{CITATION:}

Kida, Naoki ... [et al]. Finite element formulation and analysis for an arterial wall with residual and active stresses. Computer Methods in Biomechanics and Biomedical

Engineering 2015, 18(11): 1143-1159

\section{ISSUE DATE:}

2015

URL:

http://hdl.handle.net/2433/235751

\section{RIGHT:}

This is an Accepted Manuscript of an article published by Taylor \& Francis in Computer Methods in Biomechanics and Biomedical Engineering on 07 February 2014, available online:

http://www.tandfonline.com/10.1080/10255842.2013.879646; This is not the published version. Please cite only the published version.; この論文は出版社版でありません。引用の際には出版社版をご確認ご利用ください。 


\title{
Finite element formulation and analysis
}

\section{for an arterial wall with residual and active stresses}

\author{
Naoki Kida, Taiji Adachi
}

Department of Biomechanics, Institute for Frontier Medical Sciences, Kyoto University

53 Kawahara-cho, Shogoin, Sakyo, Kyoto 606-8507, Japan

Corresponding author:

Taiji Adachi, Ph.D., Professor

E-mail: adachi@frontier.kyoto-u.ac.jp

Tel: $+81-75-751-4853$

Fax: +81-75-751-4853

Submitted to Computer Methods in Biomechanics and Biomedical Engineering January 4, 2014 


\begin{abstract}
In this study, for predicting arterial function and pathogenesis from a mechanical viewpoint, we develop a continuum mechanical model of an arterial wall that embodies residual and active stresses following a traditional anisotropic passive constitutive law. The residual and active stresses are incorporated into finite element methods based on a two-field variational principle described in the Lagrangian form. The linearization of nonlinear weak-form equations derived from this variational principle is then described for developing an original finite element algorithm. Numerical simulations reveal the following: (i) residual stresses lead to a reduction in stress gradient regardless of the magnitude of external load; (ii) active stresses help homogenize stress distribution under physiological external load, but this homogeneity collapses under pathological external load; (iii) when residual and active stresses act together, the effect of the residual stresses is relatively obscured by that of the active stresses. We conclude that residual stresses have minor but persistent mechanical effects on the arterial wall under both physiological and pathological external loads, active stresses play an important role in the physiological functions and pathogenesis of arteries, and the mechanical effect of residual stresses is dependent on the presence/absence of active stresses.
\end{abstract}

\title{
Keywords
}

Artery, Residual stress, Active stress, Hyperelasticity, Nonlinear finite element analysis, Computational biomechanics 


\section{Introduction}

Arteries are important biological soft tissues that conduct blood flow and facilitate the exchange of oxygen/carbon dioxide, nutrients, and waste products between blood and tissues throughout the body. These biological activities are decreased by pathological structural changes, such as atherosclerosis or arterial aneurysm. Recent advances in molecular biology have emphasized the importance of mechanical factors in regulating the structure and function of biological tissues. Therefore, it is important to develop a solid mechanics-based arterial model and to analyze it through numerical simulations. In the field of clinical medicine, the combination of such a mechanical model and analysis could be used for evaluating physiological functions, predicting pathogenesis, and improving disease treatment. In particular, the stress distribution obtained through mechanical analysis will provide useful information about the location of pathological changes.

Like other biological soft tissues, arteries undergo finite deformations. Their mechanical behavior is highly nonlinear, anisotropic, inhomogeneous, and incompressible in the physiological state. Quantifying the mechanical stress field is equivalent to solving complex boundary-value problems with these characteristics on arbitrary three-dimensional geometries. A numerical approach using finite element methods is utilized to calculate the deformation and stress field for these complex boundary-value problems (Weiss et al., 1996; Holzapfel et al., 2002). 
In modeling the mechanical behaviors of an arterial wall, numerous constitutive laws have been proposed and evaluated (Holzapfel et al., 2000; Schröder et al., 2005). However, most such laws describe passive mechanical responses, whereas, biological arterial walls are subjected to residual and active stresses in vivo (Humphrey et al., 2002). As is well known, residual stresses decrease the maximum circumferential stresses under physiological loading, while active stresses alter the wall diameter and thickness for regulating local blood flow and altering stress distribution throughout the wall. For evaluating the mechanical behaviors of arterial walls, residual and active stresses should be formulated in a form suitable for finite element analysis. Thus far, despite the importance of these stresses, many studies have individually considered residual stress (Vaishnav et al., 1987; Van Dyke et al., 2002; Alastrué et al., 2007; Bustamante et al., 2010) and active stress (Rachev et al., 1999; Zulliger et al., 2004; Murtada et al., 2010; Stålhand et al., 2011; Schmitz et al., 2011) under physiological boundary conditions. Analyses considering the effects of both these two stresses under pathological boundary conditions are lacking. Given that the presence or absence of residual stresses could alter the magnitude of any generated active stress, incorporating both these two stresses into the model is important. Furthermore, stress analysis with the model including both residual and active stresses under pathological boundary conditions would help elucidate arterial pathogenesis.

The objectives of this study are as follows: 1) developing a continuum mechanical model 
of an arterial wall that considers both residual and active stresses in addition to the traditional passive mechanical response; 2) examining the effects of residual and active stresses on arterial pathogenesis. To this end, we calculate stress distributions under the pathological boundary conditions by performing finite element analysis on a model that incorporates both residual and active stresses. The influence of residual stresses in the arterial wall is implemented as a deformation gradient tensor derived from the opening angle experimental method (Vaishnav et al., 1987). To model active mechanical behaviors, we adopt a phenomenological strain-energy function proposed by Rachev et al. (1999) that captures the interaction between mechanical stretch and myogenic contraction. In finite element analysis of finite deformations, weak-form equations equivalent to the equations of equilibrium and kinematic boundary conditions are nonlinear and must be linearized before they are solved. Linarization involves the derivation of the fourth-order elasticity tensor, which characterizes the material properties; this key process is described in detail. The effects of residual and active stresses on the arterial wall are investigated by finite element analysis under both physiological and pathological external pressure loadings. Numerical simulations demonstrating the critical role of active stresses in pathogenesis, such as thickening of an arterial wall in the hypertensive state, are presented. 


\section{Continuum mechanical basis and variational formu-}

\section{lation for finite element implementation}

Finite element methods are widely used to analyze the nonlinear continuum mechanics of arteries. This section gives a minimal background for the finite element analysis developed in this paper. It outlines the basic kinematics, constitutive laws, and the variational formulation of a two-field energy functional in Lagrangian form.

\subsection{Deformation and constitutive laws}

The position of the particle in the reference configuration $\Omega_{0} \subset \mathbf{R}^{3}$ is denoted as $\boldsymbol{X}$. In the current configuration $\Omega \subset \mathbf{R}^{3}$, the position of the same particle is denoted as $\boldsymbol{x}$, and we assume that a bijection $\varphi: \Omega_{0} \rightarrow \Omega$ exists such that $\boldsymbol{x}=\varphi(\boldsymbol{X})$. The deformation gradient $\boldsymbol{F}$ with Jacobian $J=\operatorname{det} \boldsymbol{F}>0$ is then defined as $\boldsymbol{F}=\partial \varphi(\boldsymbol{X}) / \partial \boldsymbol{X}$, and the right CauchyGreen and Green-Lagrange strain tensors are defined as $\boldsymbol{C}=\boldsymbol{F}^{\mathrm{T}} \boldsymbol{F}$ and $\boldsymbol{E}=1 / 2(\boldsymbol{C}-\boldsymbol{I})$ respectively, where $\boldsymbol{I}$ is a second-order unit tensor. The deformation gradient tensor $\boldsymbol{F}$ is split into two components as

$$
\boldsymbol{F}=J^{1 / 3} \overline{\boldsymbol{F}}
$$

where the overbar $\left(^{-}\right)$denotes the isochoric contributions of certain physical quantities. The $J^{1 / 3} \boldsymbol{I}$ term is associated with volumetric deformations, while $\overline{\boldsymbol{F}}$ is associated with isochoric 
deformations of the material. The isochoric right Cauchy-Green and Green-Lagrange strain tensors are defined as $\overline{\boldsymbol{C}}=\overline{\boldsymbol{F}}^{\mathrm{T}} \overline{\boldsymbol{F}}$ and $\overline{\boldsymbol{E}}=1 / 2(\overline{\boldsymbol{C}}-\boldsymbol{I})$, respectively (Holzapfel, 2000).

We consider hyperelastic materials that postulate the existence of a strain-energy function $\Psi$ (defined per unit reference volume). The second Piola-Kirchhoff stress tensor $\boldsymbol{S}$ is derived from the strain-energy function $\Psi(\boldsymbol{C})$ as

$$
\boldsymbol{S}=2 \frac{\partial \Psi(\boldsymbol{C})}{\partial \boldsymbol{C}}
$$

In the representation theorem for invariants, an anisotropic hyperelastic material reinforced by two families of fibers is expressed in terms of nine invariants $I_{1}, \cdots, I_{9}$ of $\boldsymbol{C}$ and structural tensors $\boldsymbol{a}_{0} \otimes \boldsymbol{a}_{0}$ and $\boldsymbol{g}_{0} \otimes \boldsymbol{g}_{0}$, where two unit vector fields $\boldsymbol{a}_{0}$ and $\boldsymbol{g}_{0}$ in the reference configuration are implemented to describe the local fiber direction (Holzapfel, 2000; Schröder et al., 2003, 2005).

Using multiplicative decomposition of the deformation gradient tensor $\boldsymbol{F}$, the strainenergy function $\Psi$ of a slightly compressible anisotropic material is assumed to be uncoupled, in which the volumetric and isochoric components are such that

$$
\Psi\left(\boldsymbol{C}, \boldsymbol{a}_{\mathbf{0}} \otimes \boldsymbol{a}_{\mathbf{0}}, \boldsymbol{g}_{\mathbf{0}} \otimes \boldsymbol{g}_{\mathbf{0}}\right)=\Psi_{\mathrm{vol}}(J)+\Psi_{\mathrm{iso}}\left(\bar{I}_{1}, \bar{I}_{2}, \cdots, \bar{I}_{9}\right),
$$

where $\bar{I}_{1}, \cdots, \bar{I}_{9}$ are the isochoric contributions of invariants $I_{1}, \cdots, I_{9}$, respectively. The 
purely volumetric part

$$
\Psi_{\mathrm{vol}}(J)=\frac{\kappa}{2}(J-1)^{2}
$$

controls the degree of incompressibility by means of the positive parameter $\kappa$ (Brink et al., 1996). And exact incompressibility means $\kappa \rightarrow \infty$.

\subsection{Variational principle of saddle type}

Let us consider the mixed variational formulation of slightly compressible finite elasticity. The boundary-value problem associated with constrained conditions, such as slightly compressible material response (i.e., $J \simeq 1$ ) can be applied to derive the variational formulation (Sussman et al., 1987; Chang et al., 1991; Brink et al., 1996). Besides the usual displacement field $\boldsymbol{u} \in U$, another additional Lagrangian multiplier field $p \in P$ is incorporated and treated as an independent variable, where $U$ and $P$ are function spaces. The two-field functional of the total potential energy is expressed as

$$
\Pi_{\mathrm{PL}}(\boldsymbol{u}, p)=\int_{\Omega_{0}}\left[p\{J(\boldsymbol{u})-1\}-\frac{p^{2}}{2 \kappa}+\Psi_{\mathrm{iso}}\left(\bar{C}, \boldsymbol{a}_{0} \otimes \boldsymbol{a}_{0}, \boldsymbol{g}_{0} \otimes \boldsymbol{g}_{0}\right)\right] d V+\Pi_{\mathrm{ext}}(\boldsymbol{u})
$$

The second term of the integrand relaxes the incompressible constraint $J=1$ included in the

first term of the integrand. The functional $\Pi_{\text {ext }}(\boldsymbol{u})$ is the potential of conservative external forces. The boundary-value problem with constraint is equivalent to finding the stationary point $(\boldsymbol{u}, p)$ of the functional $\Pi_{\mathrm{PL}}(\boldsymbol{u}, p)$ over the set $U \times P$. In other words, we have to solve 
the saddle point problem

$$
\inf _{\boldsymbol{u} \in U} \sup _{p \in P} \Pi_{\mathrm{PL}}(\boldsymbol{u}, p)
$$

Therefore, the first variations of the functional $\Pi_{\mathrm{PL}}(\boldsymbol{u}, p)$ with respect to $\boldsymbol{u}$ and $p$ vanish for arbitrary functions $\delta \boldsymbol{u} \in U_{0}$ and $\delta p \in P$, respectively (Brink et al., 1996; Rüter et al., 2000). The set $U_{0}$ is a function space where $\delta \boldsymbol{u}=0$ on the Dirichlet boundary. The following weak-form equations are obtained from the chain rule: Find $(\boldsymbol{u}, p) \in U \times P$ such that for all $(\delta \boldsymbol{u}, \delta p) \in U_{0} \times P$,

$$
\begin{aligned}
\mathrm{D} \boldsymbol{u} \Pi_{\mathrm{PL}}(\boldsymbol{u}, p) \cdot \delta \boldsymbol{u} & =\int_{\Omega_{0}}\left(J p \boldsymbol{C}^{-1}+2 \frac{\partial \Psi_{\text {iso }}\left(\overline{\boldsymbol{C}}, \boldsymbol{a}_{0} \otimes \boldsymbol{a}_{0}, \boldsymbol{g}_{0} \otimes \boldsymbol{g}_{0}\right)}{\partial \boldsymbol{C}}\right): \delta \boldsymbol{E} d V-L_{\mathrm{ext}}(\delta \boldsymbol{u}) \\
& =0 \\
& \mathrm{D}_{p} \Pi_{\mathrm{PL}}(\boldsymbol{u}, p) \cdot \delta p=\int_{\Omega_{0}}\left(J-1-\frac{p}{\kappa}\right) \delta p d V=0
\end{aligned}
$$

where $L_{\text {ext }}(\delta \boldsymbol{u})$ is the first variation of the potential of conservative external forces $\Pi_{\text {ext }}(\boldsymbol{u})$ and $\delta \boldsymbol{E}$ is the first variation of $\boldsymbol{E}$ with respect to the displacement field $\boldsymbol{u}$. If the external forces are pressure loads which are deformation dependent and not conservative, then $L_{\text {ext }}(\delta \boldsymbol{u})$ is expressed as

$$
L_{\mathrm{ext}}(\delta \boldsymbol{u})=-p_{\mathrm{ext}} \int_{\partial \Omega_{\mathrm{N}}} \boldsymbol{n} \cdot \delta \boldsymbol{u} d s
$$

where $\boldsymbol{n}=\boldsymbol{n}(\boldsymbol{x})$ is the outward unit vector perpendicular to the pressure load surface $\partial \Omega_{\mathrm{N}}$, $p_{\text {ext }}$ is a prescribed constant pressure, and $d s$ denotes an infinitesimal surface area in the 
current configuration.

In Eq. (7a), $2 \partial \Psi_{\text {iso }}\left(\overline{\boldsymbol{C}}, \boldsymbol{a}_{0} \otimes \boldsymbol{a}_{0}, \boldsymbol{g}_{0} \otimes \boldsymbol{g}_{0}\right) / \partial \boldsymbol{C} \equiv \boldsymbol{S}_{\text {iso }}$ and $J p \boldsymbol{C}^{-1} \equiv \boldsymbol{S}_{\text {vol }}$ are the isochoric and volumetric second Piola-Kirchhoff stress tensors, respectively.

Weak-form Eqs. (7a) and (7b) are generally nonlinear in the unknown fields $\boldsymbol{u}$ and $p$. To implement these equations in mixed finite element methods, they must be linearized and solved by the Newton-Raphson iterative method. Since linearization is one of the important procedures in finite element coding and contains the fourth-order elasticity tensor that characterizes material properties, it is presented for the weak-form Eqs. (7a) and (7b) in Appendix A.

\section{Continuum mechanical formulation of an arterial wall}

In this section, we describe the mechanical properties (histological structure, residual stress, and active stress) of the arterial wall. Using a continuum mechanics approach, we develop and formulate these mechanical properties in a form suitable for finite element analysis. The arterial wall is considered as a slightly compressible thick-walled cylindrical tube, which is the simplified shape of a non-diseased artery. 


\subsection{Strain-energy function for the passive response}

Many biological soft tissues are relatively insensitive to the rate at which strain is applied and reach a preconditioned state after repeated loading. For this reason, soft tissues are considered as pseudoelastic and are modeled as hyperelastic materials (Fung et al., 1979; Weiss et al., 1996; Itskov et al., 2006).

We adopt the passive mechanical model proposed by Holzapfel et al. (2004), which accounts for the histological structure of the artery and whose results are consistent with experiment. In this model, the arterial wall is treated as a two-layer (comprising inner and outer layers) thick-walled cylindrical tube. Each layer is composed of an isotropic material and two families of collagen fibers helically wound around the arterial axis, which characterize the anisotropy, as indicated in Fig. 1. Fiber contribution to the strain-energy function is modeled using a pair of preferred directional unit vectors $\boldsymbol{a}_{0, j}$ and $\boldsymbol{g}_{0, j}$, where $j$ signifies one of in and out. In the local covariant basis of the cylindrical coordinate system $\left\{\boldsymbol{e}_{R}, \boldsymbol{e}_{\Theta}, \boldsymbol{e}_{Z}\right\}$, $\boldsymbol{a}_{0, j}$ and $\boldsymbol{g}_{0, j}$ take the following forms:

$$
\begin{aligned}
& \boldsymbol{a}_{0, j}=0 \boldsymbol{e}_{R}+\cos \eta_{j} \boldsymbol{e}_{\Theta}+\sin \eta_{j} \boldsymbol{e}_{Z}, \\
& \boldsymbol{g}_{0, j}=0 \boldsymbol{e}_{R}+\cos \eta_{j} \boldsymbol{e}_{\Theta}-\sin \eta_{j} \boldsymbol{e}_{Z},
\end{aligned}
$$

where $\eta_{j}, j=$ in, out are the angles between the fibers and the circumferential direction, as 
indicated in Fig. 1. The isochoric term of this (passive) strain-energy function depends on three invariants $\bar{I}_{1}, \bar{I}_{4}$, and $\bar{I}_{6}$ and is given by

$$
\Psi_{\text {pas,iso }}\left(\overline{\boldsymbol{C}}, \boldsymbol{a}_{0} \otimes \boldsymbol{a}_{0}, \boldsymbol{g}_{0} \otimes \boldsymbol{g}_{0}\right)=c_{e}\left(\bar{I}_{1}-3\right)+\frac{c_{1}}{2 c_{2}} \sum_{i=4,6}\left[\exp \left\{c_{2}\left(\bar{I}_{i}-1\right)^{2}\right\}-1\right]
$$

where $c_{e}>0, c_{1}>0$, and $c_{2}>0$ are material parameters. The first term on the right hand side of Eq. (10) is an isotropic contribution that depends on invariant $\bar{I}_{1}$, expressed as $\operatorname{tr} \overline{\boldsymbol{C}}$. The second anisotropic term on the right hand side of this equation contributes only when the fibers are extended, i.e., when $\bar{I}_{4}>1$ or $\bar{I}_{6}>1$, where $\bar{I}_{4}=\boldsymbol{a}_{0} \cdot \overline{\boldsymbol{C}} \boldsymbol{a}_{0}$ and $\bar{I}_{6}=\boldsymbol{g}_{0} \cdot \overline{\boldsymbol{C}} \boldsymbol{g}_{0}$.

\subsection{Residual deformation and opening angle method}

Residual stresses are present in many biological tissues, such as skeletal ligaments (Weiss et al., 2005), airways, or blood vessels. For the existence of residual stresses, arteries are not stress free even in the absence of applied loading. When an artery is transversely cut and excised from the body as a ring segment (termed the closed load-free state; see Fig. 2(a)) and then cut along its radius, it releases this stress by springing into an open segment form (termed the opened up stress-free state; see Fig. 2(b)) (Vaishnav et al., 1987). This phenomenon shows that the inner wall is compressed whereas the outer wall is circumferentially tensioned.

A common measure of the residual stresses in the closed load-free state of arterial segment is the opening angle method. Residual deformation and stresses are calculated under the 
following assumptions:

1. An arterial segment is axially symmetric (more specifically, circular) and incompressible.

2. The strain and stress fields are uniform in the axial direction.

3. Once it has been radially cut, the arterial segment is stress-free.

4. The residual deformation gradient and residual stress tensors in a closed load-free arterial ring segment are equivalent to those in a load-free artery in vivo.

To determine the residual deformation gradient $\boldsymbol{F}_{\text {res }}$, it is necessary to know the crosssectional geometry of both closed load-free (stressed) state and opened up stress-free state, including the opening angle $\alpha$. In cylindrical coordinates, the components of the local covariant bases of the closed load-free state and opened up stress-free states are $(R, \Theta, Z)$ and $\left(R^{0}, \Theta^{0}, Z^{0}\right)$, respectively (see Fig. 2(a) and $\left.2(\mathrm{~b})\right)$. On account of the cylindrical symmetry, the principal directions coincide with the axial directions $\left\{\boldsymbol{e}_{R}, \boldsymbol{e}_{\Theta}, \boldsymbol{e}_{Z}\right\}$, and the residual deformation gradient tensor $\boldsymbol{F}_{\text {res }}$ with respect to $\left\{\boldsymbol{e}^{R^{0}}, \boldsymbol{e}^{\Theta^{0}}, \boldsymbol{e}^{Z^{0}}\right\}$ and $\left\{\boldsymbol{e}_{R}, \boldsymbol{e}_{\Theta}, \boldsymbol{e}_{Z}\right\}$ is given by

$$
\boldsymbol{F}_{\mathrm{res}}=\lambda_{\mathrm{res}, R}(R) \boldsymbol{e}_{R} \otimes \boldsymbol{e}^{R^{0}}+\lambda_{\mathrm{res}, \Theta}(R) \boldsymbol{e}_{\Theta} \otimes \boldsymbol{e}^{\Theta^{0}}+\lambda_{\mathrm{res}, Z} \boldsymbol{e}_{Z} \otimes \boldsymbol{e}^{Z^{0}}
$$

where the principal residual stretches $\lambda_{\text {res }, R}(R), \lambda_{\text {res }, \Theta}(R)$, and $\lambda_{\text {res, } Z}(R)$ are the functions of a single variable, $R$. This equation plays an important role in deriving the closed load-free 
state in an arterial wall.

We next seek the principal residual stretches $\lambda_{\text {res }, R}(R), \lambda_{\text {res }, \Theta}(R)$, and $\lambda_{\text {res }, Z}(R)$. Let the inner radii, the outer radii, and the axial lengths of the opened up stress-free state be $R_{\text {in }}^{0}$, $R_{\text {out }}^{0}$, and $L^{0}$ respectively, and define the respective closed load-free state variables as $R_{\text {in }}$, $R_{\text {out }}$, and $L$ (Fig. 2(b) and 2(a)). The radii $R_{\text {in }}, R_{\text {out }}, R_{\text {in }}^{0}, R_{\text {out }}^{0}$, and the opening angle $\alpha$ are measurable quantities. From the incompressibility assumption and the boundary conditions of the $Z$-surface $\sigma^{Z Z}(R)=0\left(R_{\text {in }}<R<R_{\text {out }}\right)$, we can write

$$
2 \pi L\left\{\left(R_{\text {out }}\right)^{2}-R_{\text {in }}^{2}\right\}=(2 \pi-\alpha) L^{0}\left\{\left(R_{\text {out }}^{0}\right)^{2}-\left(R_{\text {in }}^{0}\right)^{2}\right\} .
$$

The residual axial stretch $\lambda_{\text {res }, Z}$ is a constant, defined as

$$
\lambda_{\text {res }, Z}=\frac{L}{L^{0}}=\frac{(2 \pi-\alpha)\left\{\left(R_{\text {out }}^{0}\right)^{2}-\left(R_{\text {in }}^{0}\right)^{2}\right\}}{2 \pi\left\{\left(R_{\text {out }}\right)^{2}-R_{\text {in }}^{2}\right\}}=\text { constant }
$$

Under the incompressibility assumption and specified boundary conditions, the radial coordinates of an arbitrary point in the closed load-free state $R$ and in the opened up stress-free state $R^{0}$ are related by

$$
\lambda_{\text {res }, Z} 2 \pi\left(R^{2}-R_{\text {in }}^{2}\right)=(2 \pi-\alpha)\left\{\left(R^{0}\right)^{2}-\left(R_{\text {in }}^{0}\right)^{2}\right\},
$$


from which $R^{0}$ is obtained as

$$
R^{0}=\sqrt{\left(R_{\mathrm{in}}^{0}\right)^{2}+\left(\frac{2 \pi}{2 \pi-\alpha}\right) \lambda_{\mathrm{res}, Z}\left\{R^{2}-\left(R_{\mathrm{in}}\right)^{2}\right\}}
$$

The radial, circumferential, and axial residual stretches $\lambda_{\text {res }, R}(R), \lambda_{\text {res }, \Theta}(R)$, and $\lambda_{\text {res }, Z}$ satisfy $\lambda_{\text {res }, R}(R) \lambda_{\text {res }, \Theta}(R) \lambda_{\text {res }, Z}=1$. In cylindrical coordinates $(R, \Theta, Z)$, these stretches are expressed as follows:

$$
\begin{gathered}
\lambda_{\mathrm{res}, R}(R)=F_{R^{0}}^{R}=\frac{\partial R}{\partial R^{0}}=\frac{\sqrt{\left(R_{\mathrm{in}}^{0}\right)^{2}+\left(\frac{2 \pi}{2 \pi-\alpha}\right) \lambda_{\mathrm{res}, Z}\left\{R^{2}-\left(R_{\mathrm{in}}\right)^{2}\right\}}}{\left(\frac{2 \pi}{2 \pi-\alpha}\right) \lambda_{\mathrm{res}, Z} R} \\
\lambda_{\mathrm{res}, \Theta}(R)=F_{\Theta^{0}}^{\Theta}=\frac{R \partial \Theta}{R^{0} \partial \Theta^{0}}=\frac{\left(\frac{2 \pi}{2 \pi-\alpha}\right) R}{\sqrt{\left(R_{\mathrm{in}}^{0}\right)^{2}+\left(\frac{2 \pi}{2 \pi-\alpha}\right) \lambda_{\mathrm{res}, Z}\left\{R^{2}-\left(R_{\mathrm{in}}\right)^{2}\right\}}} \\
\lambda_{\mathrm{res}, Z}=\text { constant. }
\end{gathered}
$$

The total deformation mapping of the stress-free configuration $\Omega_{\mathrm{sf}}$ onto the current configuration $\Omega$ is defined as $\varphi \circ \varphi_{\text {res }}$, where $\varphi_{\text {res }}$ is a bijection that maps points of $\Omega_{\text {sf }}$ onto the points of the reference configuration $\Omega_{0}$ and $\varphi$ is a bijection that maps points of $\Omega_{0}$ onto points of $\Omega$. Analogously, the total deformation gradient tensor $\boldsymbol{F}_{\text {total }}$ of $\Omega_{\text {sf }}$ onto $\Omega$ is defined as the dot product of two second-order tensors $\boldsymbol{F}$ and $\boldsymbol{F}_{\text {res }}$, i.e.,

$$
\boldsymbol{F}_{\text {total }}=\boldsymbol{F} \boldsymbol{F}_{\text {res }}
$$


where $\boldsymbol{F}$ and $\boldsymbol{F}_{\text {res }}$ are deformation gradient tensors of $\Omega_{0}$ onto $\Omega$ and of $\Omega_{\text {res }}$ onto $\Omega_{0}$, respectively.

Remark 1. The calculation scheme for determining residual deformation gradient tensor $\boldsymbol{F}_{\text {res }}$ using the opening angle method can be applied to incompressible and axisymmetric cylindrical arterial segments only and requires that at least two of the diagonal components of $\boldsymbol{F}_{\text {res }}$ be specified.

Remark 2. When using Eq. (15), if all components of the residual deformation gradient tensor $\boldsymbol{F}_{\text {res }}$ are known using some methods, we can analyze the mechanical behavior of arterial segments considering the effect of residual stress regardless of the non-diagonal components' values.

\subsection{Strain-energy function for active stress generation}

Active stresses are present in many biological tissues, such as skeletal muscles, airways (Wang et al., 2008), or blood vessels. In an artery, vascular smooth muscle cells generate active stresses. Before presenting the active constitutive law for an arterial wall, we describe the smooth muscle cells that generate active stresses together with their working conditions.

Smooth muscle cells are mainly circumferentially aligned and are modeled using a preferred directional unit vector $\boldsymbol{m}_{0}$ at point $\boldsymbol{X} \in \Omega_{0}$, as indicated in Fig. 1. Similar to Eqs. 
(9a) and (9b), the directional vector $\boldsymbol{m}_{0}$ in the Rachev model takes the following form:

$$
\boldsymbol{m}_{0}=0 \boldsymbol{e}_{R}+1 \boldsymbol{e}_{\Theta}+0 \boldsymbol{e}_{Z}
$$

Deformed smooth muscle cells at the associated points $\boldsymbol{x} \in \Omega$ are defined by a directional unit vector $\boldsymbol{m}$. The vectors $\boldsymbol{m}_{0}$ and $\boldsymbol{m}$ are related by $\overline{\boldsymbol{F}} \boldsymbol{m}_{0}=\bar{\lambda}_{\mathrm{m}} \boldsymbol{m}$, where $\bar{\lambda}_{\mathrm{m}}$ is the isochoric stretch of smooth muscle cells.

For the active stress generator, we consider the following assumptions.

1. Smooth muscle cells are uniformly distributed throughout the arterial wall.

2. Smooth muscle cells expand and contract in concert with their surrounding tissue. Assuming that residual stresses exist, the isochoric smooth muscle stretch $\bar{\lambda}_{\mathrm{m}}$ is defined with respect to the stress-free state (Fig. 2(b)). Conversely, the effect of residual stresses is neglected, $\bar{\lambda}_{\mathrm{m}}$ is defined with respect to the load-free state (Fig. 2(a)).

3. Smooth muscle cells can generate active stress only when $\bar{\lambda}_{\mathrm{m}}$ lies within some finite interval, i.e., $\lambda_{0}<\bar{\lambda}_{\mathrm{m}}<\lambda_{\max }$.

4. The passive response of smooth muscle cells is negligible because its effect is sufficiently small relative to other arterial responses.

5. The total Cauchy stress tensor is the sum of the passive and active Cauchy stress 
tensors $\left(\boldsymbol{\sigma}_{\text {pas }}\right.$ and $\boldsymbol{\sigma}_{\text {act }}$, respectively).

$$
\boldsymbol{\sigma}=\boldsymbol{\sigma}_{\mathrm{pas}}+\boldsymbol{\sigma}_{\mathrm{act}}
$$

In modeling active stresses, we adopt the phenomenological model proposed by Rachev et al. (1999). This model is based on the concept that embedded muscle fibers self-actively expand or contract in the direction of $\boldsymbol{m}_{0}$. The isochoric contribution of the active strainenergy function $\Psi_{\text {act,iso }}$, which depends on $\bar{\lambda}_{\mathrm{m}}$, is given by

$$
\Psi_{\text {act,iso }}\left(\bar{\lambda}_{\mathrm{m}}\right)=A\left\{\bar{\lambda}_{\mathrm{m}}+\frac{1}{3} \frac{\left(\lambda_{\max }-\bar{\lambda}_{\mathrm{m}}\right)^{3}}{\left(\lambda_{\max }-\lambda_{0}\right)^{2}}\right\}
$$

where $\lambda_{\max }$ is the stretch at which active stress generating capability is maximized, $\lambda_{0}$ is the stretch at which active stress generation ceases, and $A$ is a material constant associated with the degree of muscle activation (which has units of stress). When $A=0 \mathrm{kPa}$, the mechanical response is purely passive and smooth muscle cells are fully relaxed. Under normal physiological conditions, smooth muscle cells can generate basal active stresses. In this state, we assume $A=50 \mathrm{kPa}$. If smooth muscle cells are fully activated (e.g., by the nervous system), $A$ is set to $100 \mathrm{kPa}$. 


\subsection{Sum of the strain-energy function}

The material behavior is described by means of total strain-energy function as

$$
\Psi=\Psi_{\mathrm{vol}}(J)+\Psi_{\mathrm{pas}, \text { iso }}\left(\overline{\boldsymbol{C}}, \boldsymbol{a}_{0} \otimes \boldsymbol{a}_{0}, \boldsymbol{g}_{0} \otimes \boldsymbol{g}_{0}\right)+\Psi_{\mathrm{act}, \mathrm{iso}}\left(\bar{\lambda}_{\mathrm{m}}\right)
$$

In finite element methods described by the total Lagrangian formulation, the second Piola-

Kirchhoff stress tensor $\boldsymbol{S}$ and fourth-order elasticity tensor $\mathbb{C}$ need to be calculated. The isochoric contributions of passive second Piola-Kirchhoff stress tensor $\boldsymbol{S}_{\text {pas,iso }}$ and fourthorder elasticity tensor $\mathbb{C}_{\text {pas,iso }}$ are presented in Appendix B and those of active terms $\boldsymbol{S}_{\text {act,iso }}$ and $\mathbb{C}_{\text {act,iso }}$ are presented in Appendix $\mathrm{C}$.

\section{Comparison of the analytical and numerical solutions}

This section verifies the finite-element numerical solution developed in this study. Numerical analysis is conducted using an in-house research code developed for analyzing geometric and material nonlinear problems. The numerical and analytical solutions are compared by deriving analytical radius-pressure relationships for the presented model.

\subsection{Derivation of analytical radius-pressure relationship}

In this subsection, the arterial segment is considered as an incompressible, axially symmetric, uniform thick-walled cylindrical tube. 
We seek an analytical radius-pressure relationship $p_{\text {ext }}=f\left(r_{\text {in }}\right)$, where $p_{\text {ext }}$ is the external pressure load acting on the inner wall of the arterial segment and $r_{\text {in }}$ is an inner radius of the deformed arterial segment. The segment is subjected to pressure loads $p_{\text {ext }}$ on the inner surface but no forced displacements occur in the axial direction $\left(\lambda_{z}=1.0\right)$. By assuming axial symmetry, the boundary-value problem is reduced to a one-dimensional problem in terms of radial position $r$.

The following points require careful considerations: first, the shear components of the passive Cauchy stress tensor vanish (i.e., $\sigma_{\text {pas }}^{r \theta}=\sigma_{\text {pas }}^{\theta z}=\sigma_{\text {pas }}^{z r}=0$ ) because the collagen fibers are symmetrically distributed in the $Z$ plane. Second, all components of the active Cauchy stress tensor vanish except the $\theta \theta$ component. Because the smooth muscle cells are assumed to be aligned in the circumferential direction only, the argument of $\sigma_{\text {act }}^{\theta \theta}$ is the circumferential stretch $\lambda_{\theta}=F_{\Theta}^{\theta}$.

The general constitutive equation for incompressible hyperelastic materials including active stresses is

$$
\boldsymbol{\sigma}=-p_{\mathrm{rs}} \boldsymbol{I}+\boldsymbol{F}\left(\frac{\partial \Psi_{\mathrm{pas}}}{\partial \boldsymbol{F}}\right)^{\mathrm{T}}+\boldsymbol{F}\left(\frac{\partial \Psi_{\mathrm{act}}}{\partial \boldsymbol{F}}\right)^{\mathrm{T}}
$$

where $-p_{\mathrm{rs}} \boldsymbol{I}$ is a reaction stress tensor, $\Psi_{\mathrm{pas}}$ is a passive strain-energy function with arguments $\boldsymbol{F}, \boldsymbol{a}_{0} \otimes \boldsymbol{a}_{0}$, and $\boldsymbol{g}_{0} \otimes \boldsymbol{g}_{0}$ (defined for $\operatorname{det} \boldsymbol{F}=1$ ), and $\Psi_{\text {act }}$ is an active strain-energy function with arguments $\lambda_{\mathrm{m}}$. The arguments of the (passive and active) strain-energy functions are omitted in this subsection for brevity. Let the the material point in cylindrical 
coordinates, inner radius, and outer radius of the closed load-free state be $(R, \Theta, Z), R_{\text {in }}$, and $R_{\text {out }}$ respectively, and denote the equivalent variables for the loaded deformed state as $(r, \theta, z), r_{\text {in }}$, and $r_{\text {out }}($ Fig. 2(a) and 2(c)). By cylindrical symmetry, the principal directions coincide with the axial directions $(r, \theta, z)$ and the deformation gradient tensor $\boldsymbol{F}$ is given in terms of $\left\{\boldsymbol{e}^{R}, \boldsymbol{e}^{\Theta}, \boldsymbol{e}^{Z}\right\}$ and $\left\{\boldsymbol{e}_{r}, \boldsymbol{e}_{\theta}, \boldsymbol{e}_{z}\right\}$ by

$$
\boldsymbol{F}=\lambda_{r}(r) \boldsymbol{e}_{r} \otimes \boldsymbol{e}^{R}+\lambda_{\theta}(r) \boldsymbol{e}_{\theta} \otimes \boldsymbol{e}^{\Theta}+\lambda_{z}(r) \boldsymbol{e}_{z} \otimes \boldsymbol{e}^{Z},
$$

where principal stretches $\lambda_{r}(r), \lambda_{\theta}(r)$, and $\lambda_{z}(r)$ are functions of $r$. Note that the reaction stress tensor $-p_{\mathrm{rs}} \boldsymbol{I}$ is also a function of $r$ alone.

We next define principal stretches $\lambda_{r}(r)$ and $\lambda_{\theta}(r)$. Assuming incompressibility and the boundary condition $\lambda_{z}=1$, we obtain

$$
R=\sqrt{\left(r^{2}-r_{\mathrm{in}}^{2}\right)+R_{\mathrm{in}}^{2}}
$$

Analogously, the outer radius $r_{\text {out }}$ of the deformed arterial segment is obtained as

$$
r_{\mathrm{out}}=\sqrt{\left(R_{\mathrm{out}}^{2}-R_{\mathrm{in}}^{2}\right)+r_{\mathrm{in}}^{2}}
$$

The radial, circumferential, and axial stretches $\lambda_{r}(r), \lambda_{\theta}(r)$, and $\lambda_{z}$ satisfy $\lambda_{r}(r) \lambda_{\theta}(r) \lambda_{z}=1$. 
In cylindrical coordinates $(r, \theta, z)$, these stretches are expressed as

$$
\begin{gathered}
\lambda_{r}(r)=F_{R}^{r}=\frac{\partial r}{\partial R}=\frac{\sqrt{\left(r^{2}-r_{\text {in }}^{2}\right)+R_{\text {in }}^{2}}}{r} \\
\lambda_{\theta}(r)=F_{\Theta}^{\theta}=\frac{r \partial \theta}{R \partial \Theta}=\frac{r}{\sqrt{\left(r^{2}-r_{\text {in }}^{2}\right)+R_{\text {in }}^{2}}} \\
\lambda_{z}=F_{Z}^{z}=1 .
\end{gathered}
$$

Because the body forces are negligible compared with the surface traction loads and internal forces, and given the geometrical and constitutive symmetry, all components of the equilibrium equations vanish except

$$
\frac{d \sigma^{r r}}{d r}+\frac{\sigma^{r r}-\sigma^{\theta \theta}}{r}=0
$$

From Eq. (25) and the following boundary conditions on the inner and outer surface of an arterial segment

$$
\sigma^{r r}\left(\mathrm{r}_{\text {out }}\right)=0, \quad \sigma^{r r}\left(\mathrm{r}_{\text {in }}\right)=-p_{\text {ext }}
$$

the external pressure load acting on an inner wall of the artery $\left(p_{\text {ext }}\right)$ is calculated as

$$
p_{\text {ext }}=-\int_{r_{\text {in }}}^{r_{\text {out }}} \frac{\sigma^{r r}-\sigma^{\theta \theta}}{r} d r
$$

Note that $p_{\text {ext }}$ is defined such that compression is positive. Substituting the cylindrical 
components of Eq. (20) into Eq. (27) we obtain

$$
p_{\text {ext }}\left(r_{\text {in }}\right)=\int_{r_{\text {in }}}^{r_{\text {out }}}\left(\lambda_{\theta} \frac{\partial \Psi_{\mathrm{pas}}}{\partial \lambda_{\theta}}+\lambda_{\theta} \frac{\partial \Psi_{\mathrm{act}}}{\partial \lambda_{\theta}}-\lambda_{r} \frac{\partial \Psi_{\mathrm{pas}}}{\partial \lambda_{r}}\right) \frac{d r}{r} .
$$

The final step in relating the pressure to radius is substituting the (passive and active) strain-energy function into Eq. (28) and integrating. The terms $\partial \Psi_{\text {pas }} / \partial \lambda_{\theta}, \lambda_{\theta} \partial \Psi_{\text {act }} / \partial \lambda_{\theta}$, and $\partial \Psi_{\text {pas }} / \partial \lambda_{r}$ in Eq. (28) are calculated in Appendix D.

In integrating Eq. (28), the following four points should be noted: first, the material constants differ between the inner and outer layers. Second, the anisotropic term in the passive strain-energy function should contribute only when the collagen fibers are extended; that is, when $I_{4}>1$ or $I_{6}>1$. Third, for smooth muscle cells to generate active stresses, their stretch $\lambda_{\mathrm{m}}$ must lie within a finite interval. Finally, because closed-form evaluation is possible only under simple constitutive laws such as the Moony-Rivlin model, the radiuspressure curve is obtained by numerical quadrature.

\subsection{Conditions of the numerical analysis}

The passive and active material constants in Eqs. (10) and (18) and the geometric data used in the model are listed in Tables 1, 2, and 3. Consistent with previous works, the axial length of the arterial segment is assumed to be equal to the inner radius in the load-free state. The mean ratio of the inner-layer and outer-layer wall thicknesses is supposed to be 
$2: 1$. The body forces are regarded as insignificant compared to surface traction loads and internal forces.

The maximum external pressure load $p_{\text {ext }}=60 \mathrm{kPa}$ with constant axial stretch $\lambda_{z}=1.0$ is applied as a boundary condition to the inner wall.

In the finite element analysis, the arterial segment is considered as a slightly compressible thick-walled cylindrical tube with axial symmetry and axial uniformity. Finite element analysis is conducted under the following conditions: (i) The arterial segment is discretized into 9 radial elements, 108 circumferential element, and 18 axial elements, and is modeled by the $Q_{1} / P_{0}$-element (i.e., trilinear displacement and constant Lagrange multiplier approximations); (ii) The penalty parameter $\kappa$ is taken as $c_{e} \times 10^{4}$; (iii) Newton-Raphson iterations continue until all normalized square norms are below $10^{-5}$; (iv) Boundary conditions are divided into 10 equal increments. Large sparse linear systems and eigenvalue problems are solved using the PARDISO solver (included in the Intel Math Kernel Library) and LAPACK routines, respectively.

Remark 3. The $Q_{1} / P_{0}$-element is the lowest order mixed approximation for a hexahedral finite element; however, it does not satisfy the discrete inf-sup condition and it exhibits the spurious Lagrange multiplier modes of a checkerboard instability (Silvester et al., 1990; Vincent et al., 1992; Chapelle et al., 1993). Conversely, the $Q_{1} / P_{0}$-elements gives good results with the use of a suitable posteriori filtering technique. The term $-p^{2} / 2 \kappa$ in Eq. (5) was 
introduced to filter the spurious Lagrange multiplier mode. In this study, we use the $Q_{1} / P_{0^{-}}$ element for discretization, since this element is one of the cheapest and most popular elements for three-dimensional problems from the computational implementation aspects.

\subsection{Verification of the numerical results}

The external pressure loads acting on an inner wall of the artery $\left(p_{\text {ext }}\right)$ as a function of deformed inner radius $\left(r_{\text {in }}\right)$ are shown in Fig. 3. Both analytical and finite element solutions are provided. The passive mechanical response $(A=0 \mathrm{kPa})$ yields strong agreement between the two solutions (Fig. 3 dashed line and symbol "o"). Under active mechanical response $(A=50 \mathrm{kPa})$, the smoothness of the graph disappears around the bifurcation point $\left(r_{\text {in }}\right.$ being a multi-variable function of $p_{\text {ext }}$ in the neighborhood of $25.0 \mathrm{kPa}$ ) and the theoretical and numerical solutions dissociate (Fig. 3 solid line and symbol " $\square$ "). By appropriately dividing the external load increments, the solutions converge. In both cases, the solution converges within three to five iterations for every load step.

\section{Numerical examples of stress distribution in the ar- terial segment}

In this section, we assume fixed approximate axial in situ stretch of the artery (1.7) and impose various external pressure loads (inner pressures) as boundary conditions. We examine 
the stress distributions in an arterial segment considering the effect of residual and/or active stresses (smooth muscle cells are in basal tone, i.e., $A=50 \mathrm{kPa}$ ). The stress distributions are computed by finite element analysis under the conditions of Section 4.2, using the same material constants and geometric data.

\subsection{Effects of physiological loading}

The Cauchy stress distribution through the arterial segment in the presence/absence of residual and/or active stresses is obtained under a physiological external pressure load $p_{\text {ext }}=13.33$ $\mathrm{kPa}$. The following four cases are considered.

- Case 1: Absence of residual and active stresses (o).

- Case 2: Presence of residual stresses; absence of active stresses $(\bullet)$.

- Case 3: Absence of residual stresses; presence of active stresses ( $\square$ ).

- Case 4: Presence of residual and active stresses

In Case 2 and Case 4, the (total) deformation gradient tensor is the dot product of the deformation gradient tensor $\boldsymbol{F}$ and the residual deformation gradient tensor $\boldsymbol{F}_{\text {res }}$ (see. Eq. (15)). In Case 3, smooth muscle stretch is defined with respect to the closed load-free state and in Case 4 with respect to the opened up stress-free state. 
Figure 4 shows the distributions of the principal Cauchy stress components $\sigma^{\theta \theta}$ and $\sigma^{z z}$ as functions of deformed radial coordinate $r$. In the circumferential direction (Fig. 4(a)), residual stress leads to reduction in the stress gradient $(\bullet)$. However, stress discontinuity persists at the interface between the inner and outer layer. With the inclusion of active stresses, the stress distribution tends to become more homogeneous ( $\square)$. When both residual and active stresses exist ( $)$, the stress distribution is similar to that of active stresses alone. Active stresses play a critical role in eliminating stress discontinuity and homogenizing the stress distribution. In the axial direction (Fig. 4(b)), residual stresses exert little effects on stress distributions $(\bullet)$. Active stresses play a small role in reducing the stress gradient but do not reduce axial stress discontinuity, because they are assumed to act in the circumferential directional alone $(\square)$. It should be noted that incorporation of active contraction in the axial direction may change this conclusion.

\section{$5.2 \quad$ Effects of pathological loading}

In the following, we focus on the Cauchy stress distributions in the circumferential direction, the assumed direction of active stresses. Figure 5 shows the distributions of the circumferential Cauchy stress component $\sigma^{\theta \theta}$ as functions of the deformed radial coordinate $r$ under various external pressure loads $p_{\text {ext }}$ (namely $13.33,16.00,20.00$, and $26.66 \mathrm{kPa}$, corresponding to normal mean blood pressure, normal systolic blood pressure, hypertension, and severe 
hypertension, respectively). Figure 5(a) shows the stress distribution under passive mechanical response, i.e., absence of residual and active stresses. High external pressure loads cause increases in both inner radius and stress gradient. Residual stresses (see Fig. 5(b)) reduce the stress gradient and maximum stress at all external pressure loads. When active stresses are presented (see Fig. 5(c)), the uniform stress distribution resulting from smooth muscle activation collapses as external pressure load increases. In the presence of both residual and active stresses (Fig. 5(d)), residual stresses are less important than active stresses in reducing the stress gradient under physiological load, although they decrease the maximum stresses at all magnitudes of external pressure load.

\subsection{Thickness of the arterial wall under hypertensive loading}

Experiments have shown that strain and stress distributions throughout the arterial wall are nearly uniform under physiological loadings (Matsumoto et al., 1996). If hypertensive loading persists, the thickness of the inner layer increases to maintain the circumferential stress around the level of normotensive loading (Fung et al., 1989).

The second of the above mentioned experimental findings is likely related to the essential role of the active stresses. To investigate this idea, we subject the arterial wall to hypertensive loading and calculate the changes in stress distribution. In response, the wall thickness of the closed load-free arterial segment increases toward the inside of the cavity, whereas the 
outer radius remains unchanged (Fig. 6). In the wall-thickening process, we suppose that smooth muscle cells are in basal tone $(A=50 \mathrm{kPa})$ and that thickness increases in the inner layer only; that is, the ratio between outer and inner radius deviates from $2: 1$. In this scenario, residual stresses are not considered, because their effect is obscured by that of active stresses when residual and active stresses act together as indicated in the previous subsection. The external pressure load $p_{\text {ext }}$ applied to the inner wall is $26.66 \mathrm{kPa}$ mimicking severe hypertension.

Suppose that the wall thickness of a normal arterial segment is 1. Under high loads, suppose that the thickness of the arterial wall has increased by factors of $1.111,1.222$, and 1.333. Figure 7 shows the circumferential Cauchy stress distributions through the deformed wall thickness as wall thickness increases. In the absence of active stresses, no reduction in transmural stress gradient is observed (Fig. 7(a)). However, presence of active stresses does reduce this gradient (Fig. $7(\mathrm{~b}))$. In clinical hypertension, when stress gradients occur in the arterial wall, the arteries afferently increase the thicknesses of their inner layer to restore the stress distributions to normotensive (physiological) levels. Smooth muscle activation plays an important role in this process. 


\section{Discussion}

Numerous mechanical models of arterial walls have been proposed and examined in previous works (Holzapfel et al., 2000; Schröder et al., 2005). These literatures, which have mainly analyzed the passive mechanical responses described by hyperelastic materials, have demonstrated the existence of large stress gradients through the arterial wall and high stresses in the innermost layer. Such stress distribution has been suggested to predispose the inner surface of an artery to arteriosclerosis (Humphrey et al., 2002). However, both residual and active stresses are present in arterial walls. Incorporating residual stresses into the stress analyses under physiological boundary conditions reduces the stress gradients (Vaishnav et al., 1987). Stress gradients are further reduced if active stresses are added (Rachev et al., 1999). Including these stresses in the analysis replicates an experimental fact: that is, the strain and stress distributions are nearly uniform throughout the arterial wall in physiological boundary conditions (Matsumoto et al., 1996). Residual and active stresses are now recognized as essential biomechanical factors.

In general, biological tissue maintains its balance by producing and removing its constituents (a process called turnover). It is widely believed that homogeneous stress distribution prevents incompatible turnover (Humphrey, 2003); that is, inhomogeneous stress distribution could induce a locally incompatibled turnover. For example, high blood pressure against the arterial wall alters the stress distribution within it. The resulting incompatible 
turnover could lead to wall thickening toward the inside of the cavity as the wall attempts to restore homogeneous stress distribution (Fung et al., 1989). In an artery, both residual and active stresses are presumed to create or reduce the stress gradients that promote pathogenesis or remodeling under external loads. Despite the non-negligible functions of these two stresses, few researchers have attempted to quantify the effects of both residual and active stresses.

In this study, for realistically predicting arterial function and pathogenesis from a mechanical viewpoint, we developed a continuum mechanical model of arterial wall that incorporates both residual and active stresses in addition to the traditional passive mechanical response. Using the developed model, we examined the effects of these stresses on the pathogenesis of an artery by calculating stress distributions under the pathological boundary conditions by performing finite element analysis. The following results were obtained: (i) residual stresses lead to a reduction in stress gradient regardless of the magnitude of external load; (ii) active stresses have a role in homogenizing the stress distribution under physiological external load, but such induced homogeneity collapses under pathological external load; (iii) when residual and active stresses act together, the effect of the residual stresses is relatively obscured by that of the active stresses. Our computer simulations replicated an experimentally determined phenomenon in hypertensive loading, that is, stress gradients in an arterial wall cause an afferent increase in wall thickness, which restores stress distributions to their physiologi- 
cal levels. From these results, we conclude that residual stresses have minor but persistent mechanical effects on the arterial wall under both physiological and pathological external loads, active stresses play an important role in the physiological functions and pathogenesis of arteries, and the mechanical effect of residual stresses is dependent on the presence or absence of active stresses.

Residual and/or active stresses are observed in many biological tissues and have an important role in their physiological functions. Studies have been conducted on the mathematical modeling and numerical analyses of the mechanical behavior of biological tissues including residual and/or active stresses, such as those of the airway (Wang et al., 2008) or skeletal ligaments (Weiss et al., 2005). As for the arterial wall, residual stresses are modeled using the opening angle method or its variants (Van Dyke et al., 2002). We chose the opening angle experimental method for incorporating residual stresses into numerical analysis, because a method for theoretically estimating the residual deformation gradient tensor of a hollow cylindrical tube artery is yet to be established. For modeling active stresses, a phenomenological strain-energy function (Rachev et al., 1999; Zulliger et al., 2004; Schmitz et al., 2011) or the mechanical model of a microstructure that generates active stresses (Murtada et al., 2010; Stålhand et al., 2011) is used. The former is straightforward to adapt to finite element analysis, but given that it is an extrapolation from experiments, this method lacks any theoretical basis. The latter model is deduced theoretically, but it is difficult to 
determine material constants and integrate the macrostructure. We chose the strain-energy function approach for incorporating active stresses into numerical analysis, because by doing so, relatively few parameters are required for determining material behavior.

Several simplifying assumptions were made in this study. First, residual stresses were assessed by the opening angle method. However, this method provides essentially twodimensional information because it assumes that an arterial segment is circular and uniform. The opening angle method is inadequate for determining three-dimensional residual stress distributions. For instance, within inhomogeneous materials, the opening angle $\alpha$ or axial stretch $\lambda_{z}$ should differ from layer to layer (Bustamante et al., 2010). In arbitrary threedimensional geometries, the experimental method should be replaced with a theoretical one that calculates the residual deformation gradient tensors. Second, we assumed that active stress generators, i.e., smooth muscle cells, are uniformly distributed in the wall, align and generate active stresses in the circumferential direction only. However, smooth muscle cells may be contained in the middle layer of the wall and are not necessarily one-dimensional; they possibly undergo multi-axial contraction and relaxation (Takamizawa et al., 1992; Humphrey et al., 2002). An improved constitutive law of the active stress generator would eliminate the stress discontinuity in inhomogeneous materials, rendering all components of the Cauchy stress tensor uniform throughout the arterial wall. Third, because there are in vivo blood flows inside the cavity of the vessel, fluid-solid interactions cannot be ignored in a computa- 
tional mechanical approach (Humphrey et al., 2002).

In future studies, we plan to develop a model that consider the above described assumptions and try to analyze patient specific geometries under complicate boundary conditions. If computational arterial models based on appropriate constitutive laws and boundary conditions are established, physiological functions could be predicted more realistically, leading to improved diagnosis or disease treatment. 


\section{Acknowledgements}

This research work is supported partly by the Funding Program for Next Generation World-

Leading Researchers (LR017) from the Ministry of Education, Culture, Sports, Science and

Technology (MEXT) in Japan. 


\section{Appendix A Linearization of weak form}

Linearization is an important process in nonlinear finite element coding. Therefore, this Appendix summarizes the linearization procedure of the weak form Eqs. (7a) and (7b) described in the variational principle of saddle type of Section 2.2. Further details and mathematical background are provided in Brink et al. (1996) and Rüter et al. (2000).

Eqs. (7a) and (7b) are linearized by the second variation of Eq. (5). For simplicity, the terms in Eqs. (7a) and (7b) are expressed in short-hand notation as

$$
\begin{gathered}
G_{u p}(\delta \boldsymbol{u})=\int_{\Omega_{0}}\left\{J p \boldsymbol{C}^{-1}+2 \frac{\partial \Psi_{\text {iso }}\left(\overline{\boldsymbol{C}}, \boldsymbol{a}_{0} \otimes \boldsymbol{a}_{0}, \boldsymbol{g}_{0} \otimes \boldsymbol{g}_{0}\right)}{\partial \boldsymbol{C}}\right\}: \delta \boldsymbol{E} d V \\
F_{u p}(\delta p)=\int_{\Omega_{0}}\left(J-1-\frac{p}{\kappa}\right) \delta p d V .
\end{gathered}
$$

Linearization is based on first-order Taylor's expansion, expressed as

$$
\begin{array}{r}
G_{u p}(\delta \boldsymbol{u})+\mathrm{D} \boldsymbol{u} G_{u p}(\delta \boldsymbol{u}) \cdot \Delta \boldsymbol{u}+\mathrm{D}_{p} G_{u p}(\delta \boldsymbol{u}) \cdot \Delta p \\
+o(\|\Delta \boldsymbol{u}\|)+o(\|\Delta p\|)=L_{\text {ext }}(\delta \boldsymbol{u}), \\
F_{u p}(\delta p)+\mathrm{D}_{\boldsymbol{u}} F_{u p}(\delta p) \cdot \Delta \boldsymbol{u}+\mathrm{D}_{p} F_{u p}(\delta p) \cdot \Delta p \\
+o(\|\Delta \boldsymbol{u}\|)+o(\|\Delta p\|)=0,
\end{array}
$$


where $\Delta(\cdot)$ denotes the linearization operator. The quantities $\Delta \boldsymbol{u}$ and $\Delta p$ denote the increment of the displacement field $\boldsymbol{u}$ and the Lagrange multiplier field $p$, respectively. The term $o(\|\Delta x\|)$, characterized by the Landau order symbol, is a small error that tends to zero faster than $\|\Delta x\| \rightarrow 0$, i.e., $\lim _{\|\Delta x\| \rightarrow 0} O(\|\Delta x\|) /\|\Delta x\| \rightarrow 0$.

By substituting Eqs. (A.1) and (A.2) into Eqs. (A.3a) and (A.3b) and omitting $o(\|\Delta \boldsymbol{u}\|)$ and $o(\|\Delta p\|)$, the linearized weak form is obtained as follows: given the current state $(\boldsymbol{u}, p) \in U \times P$, find increments $(\Delta \boldsymbol{u}, \Delta p) \in U_{0} \times P$ such that for all $(\delta \boldsymbol{u}, \delta p) \in U_{0} \times P$,

$$
\begin{gathered}
a_{u p}(\Delta \boldsymbol{u}, \delta \boldsymbol{u})+b_{u p}(\Delta p, \delta \boldsymbol{u})=L_{\mathrm{ext}}(\delta \boldsymbol{u})-G_{u p}(\delta \boldsymbol{u}), \\
b_{u p}(\delta p, \Delta \boldsymbol{u})+c_{u p}(\Delta p, \delta p)=-F_{u p}(\delta p),
\end{gathered}
$$

where

$$
\begin{aligned}
& a_{u p}(\Delta \boldsymbol{u}, \delta \boldsymbol{u}) \equiv \mathrm{D} \boldsymbol{u} G_{u p}(\delta \boldsymbol{u}) \cdot \Delta \boldsymbol{u} \\
&= \int_{\Omega_{0}}\left(J p \boldsymbol{C}^{-1}+2 \frac{\partial \Psi_{\mathrm{iso}}\left(\overline{\boldsymbol{C}}, \boldsymbol{a}_{0} \otimes \boldsymbol{a}_{0}, \boldsymbol{g}_{0} \otimes \boldsymbol{g}_{0}\right)}{\partial \boldsymbol{C}}\right):(\operatorname{Grad} \Delta \boldsymbol{u})^{\mathrm{T}} \mathrm{Grad} \delta \boldsymbol{u} \\
&+\delta \boldsymbol{E}:\left(2 p \frac{\partial J \boldsymbol{C}^{-1}}{\partial \boldsymbol{C}}+4 \frac{\partial^{2} \Psi_{\mathrm{iso}}\left(\overline{\boldsymbol{C}}, \boldsymbol{a}_{0} \otimes \boldsymbol{a}_{0}, \boldsymbol{g}_{0} \otimes \boldsymbol{g}_{0}\right)}{\partial \boldsymbol{C} \partial \boldsymbol{C}}\right): \Delta \boldsymbol{E} d V \\
& b_{u p}(\Delta p, \delta \boldsymbol{u}) \equiv \mathrm{D}_{p} G_{u p}(\delta \boldsymbol{u}) \cdot \Delta p \\
&=\int_{\Omega_{0}} J \Delta p \boldsymbol{C}^{-1}: \delta \boldsymbol{E} d V=\int_{\Omega_{0}} J \Delta p \operatorname{div} \delta \boldsymbol{u} d V
\end{aligned}
$$




$$
\begin{aligned}
& b_{u p}(\delta p, \Delta \boldsymbol{u}) \equiv \mathrm{D}_{\boldsymbol{u}} F_{u p}(\delta p) \cdot \Delta \boldsymbol{u} \\
&=\int_{\Omega_{0}} J \delta p \boldsymbol{C}^{-1}: \Delta \boldsymbol{E} d V=\int_{\Omega_{0}} J \delta p \operatorname{div} \Delta \boldsymbol{u} d V, \\
& c_{u p}(\delta p, \Delta p) \equiv \mathrm{D}_{p} F_{u p}(\delta p) \cdot \Delta p=-\int_{\Omega_{0}} \frac{\delta p \Delta p}{\kappa} d V .
\end{aligned}
$$

Here we use bilinear forms $a_{u p}: U_{0} \times U_{0} \rightarrow \mathbf{R}, b_{u p}: P \times U_{0} \rightarrow \mathbf{R}, c_{u p}: P \times P \rightarrow \mathbf{R}$ and linear forms $L_{\text {ext }}: U_{0} \rightarrow \mathbf{R}, G_{u p}: U_{0} \rightarrow \mathbf{R}, F_{u p}: P \rightarrow \mathbf{R}$.

In Eq. (A.5), $4 \partial^{2} \Psi_{\text {iso }}\left(\overline{\boldsymbol{C}}, \boldsymbol{a}_{0} \otimes \boldsymbol{a}_{0}, \boldsymbol{g}_{0} \otimes \boldsymbol{g}_{0}\right) / \partial \boldsymbol{C} \partial \boldsymbol{C} \equiv \mathbb{C}_{\text {iso }}$ and $2 p \partial\left(J \boldsymbol{C}^{-1}\right) / \partial \boldsymbol{C} \equiv \mathbb{C}_{\mathrm{vol}}$ are the isochoric and volumetric fourth-order elasticity tensors, respectively. These elasticity tensors embody constitutive laws, and thus play an important role in nonlinear finite element methods. 


\section{Appendix B The isochoric contributions of passive sec-}

\section{ond Piola-Kirchhoff stress tensor $S_{\text {pas,iso }}$ and fourth-order elasticity tensor $\mathbb{C}_{\text {pas,iso }}$}

Finite element codes described by the total Lagrangian formulation require calculation of the second Piola-Kirchhoff stress tensor $\boldsymbol{S}$ and fourth-order elasticity tensor $\mathbb{C}$. This Appendix derives the isochoric contributions of passive second Piola-Kirchhoff stress tensor $\boldsymbol{S}_{\text {pas,iso }}$ and

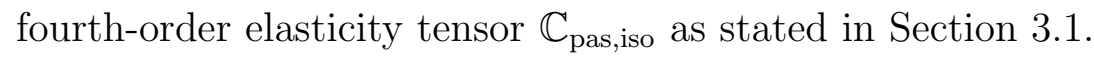

The isochoric contribution of the passive second Piola-Kirchhoff stress tensor $\boldsymbol{S}_{\text {pas,iso }}$ is given by

$$
\begin{aligned}
\boldsymbol{S}_{\mathrm{pas}, \text { iso }} & =\frac{\partial \Psi_{\mathrm{pas}, \text { iso }}\left(\bar{I}_{1}, \bar{I}_{4}, \bar{I}_{6}\right)}{\partial \boldsymbol{C}} \\
& =2 \frac{\partial \Psi_{\mathrm{pas}, \text { iso }}\left(\bar{I}_{1}, \bar{I}_{4}, \bar{I}_{6}\right)}{\partial \overline{\boldsymbol{C}}}: \frac{\partial \overline{\boldsymbol{C}}}{\partial \boldsymbol{C}} \\
& =2 \frac{\partial \Psi_{\mathrm{pas}, \text { iso }}\left(\bar{I}_{1}, \bar{I}_{4}, \bar{I}_{6}\right)}{\partial \overline{\boldsymbol{C}}}: J^{-2 / 3}\left(\mathbb{I}-\frac{1}{3} \boldsymbol{C} \otimes \boldsymbol{C}^{-1}\right) \\
& =J^{-2 / 3} \mathbb{P}: 2 \frac{\partial \Psi_{\mathrm{pas}, \text { iso }}\left(\bar{I}_{1}, \bar{I}_{4}, \bar{I}_{6}\right)}{\partial \overline{\boldsymbol{C}}}=J^{-2 / 3} \mathbb{P}: \overline{\boldsymbol{S}}_{\mathrm{pas}} .
\end{aligned}
$$

where $\mathbb{P}$ is the fourth-order material projection tensor defined as

$$
\mathbb{P}=\mathbb{I}-\frac{1}{3} \boldsymbol{C}^{-1} \otimes \boldsymbol{C},
$$


and $\mathbb{I}$ is a fourth-order unit tensor. The passive fictitious second Piola-Kirchhoff stress tensor $\overline{\boldsymbol{S}}_{\mathrm{pas}}$ is defined as

$$
\begin{aligned}
\overline{\boldsymbol{S}}_{\text {pas }} \equiv & 2 \frac{\partial \Psi_{\text {pas,iso }}\left(\bar{I}_{1}, \bar{I}_{4}, \bar{I}_{6}\right)}{\partial \overline{\boldsymbol{C}}} \\
= & c_{e} \boldsymbol{I}+c_{1}\left(\bar{I}_{4}-1\right)\left[\exp \left\{c_{2}\left(\bar{I}_{4}-1\right)^{2}\right\}\right] \boldsymbol{a}_{0} \otimes \boldsymbol{a}_{0} \\
& +c_{1}\left(\bar{I}_{6}-1\right)\left[\exp \left\{c_{2}\left(\bar{I}_{6}-1\right)^{2}\right\}\right] \boldsymbol{g}_{0} \otimes \boldsymbol{g}_{0}
\end{aligned}
$$

Here we have used the following relationships:

$$
\frac{\partial \bar{I}_{1}}{\partial \overline{\boldsymbol{C}}}=\boldsymbol{I}, \quad \frac{\partial \bar{I}_{4}}{\partial \overline{\boldsymbol{C}}}=\boldsymbol{a}_{0} \otimes \boldsymbol{a}_{0}, \quad \frac{\partial \bar{I}_{6}}{\partial \overline{\boldsymbol{C}}}=\boldsymbol{g}_{0} \otimes \boldsymbol{g}_{0}
$$

where $\boldsymbol{I}$ is a second-order unit tensor.

The isochoric contribution of the passive fourth-order elasticity tensor $\mathbb{C}_{\text {pas,iso }}$ is given by (see Holzapfel (2000))

$$
\begin{aligned}
\mathbb{C}_{\text {pas }, \text { iso }}= & 2 \frac{\partial \boldsymbol{S}_{\text {pas }, \text { iso }}}{\partial \boldsymbol{C}}=4 \frac{\partial^{2} \Psi_{\text {pas }, \text { iso }}\left(\bar{I}_{1}, \bar{I}_{4}, \bar{I}_{6}\right)}{\partial \boldsymbol{C} \partial \boldsymbol{C}} \\
= & \mathbb{P}: 2 J^{-4 / 3} \frac{\partial \overline{\boldsymbol{S}}_{\mathrm{pas}}}{\partial \overline{\boldsymbol{C}}}: \mathbb{P}^{\mathrm{T}}+\frac{2}{3}\left(J^{-2 / 3} \overline{\boldsymbol{S}}_{\mathrm{pas}}: \boldsymbol{C}\right) \tilde{\mathbb{P}} \\
& -\frac{2}{3}\left(\boldsymbol{C}^{-1} \otimes \boldsymbol{S}_{\mathrm{pas}, \text { iso }}+\boldsymbol{S}_{\text {pas }, \text { iso }} \otimes \boldsymbol{C}^{-1}\right)
\end{aligned}
$$


Here we have used the fourth-order modified material projection tensor

$$
\tilde{\mathbb{P}}=-\frac{\partial \boldsymbol{C}^{-1}}{\partial \boldsymbol{C}}-\frac{1}{3} \boldsymbol{C}^{-1} \otimes \boldsymbol{C}^{-1}
$$

and the notation

$$
\begin{aligned}
\frac{\partial \overline{\boldsymbol{S}}_{\mathrm{pas}}}{\partial \overline{\boldsymbol{C}}}= & \left\{2 c_{2}\left(\bar{I}_{4}-1\right)^{2}+1\right\} c_{1} \exp \left\{c_{2}\left(\bar{I}_{4}-1\right)^{2}\right\} \boldsymbol{a}_{0} \otimes \boldsymbol{a}_{0} \otimes \boldsymbol{a}_{0} \otimes \boldsymbol{a}_{0} \\
& +\left\{2 c_{2}\left(\bar{I}_{6}-1\right)^{2}+1\right\} c_{1} \exp \left\{c_{2}\left(\bar{I}_{6}-1\right)^{2}\right\} \boldsymbol{g}_{0} \otimes \boldsymbol{g}_{0} \otimes \boldsymbol{g}_{0} \otimes \boldsymbol{g}_{0} .
\end{aligned}
$$

When implementing these equations in finite element code, the cylindrical components of the second- and fourth-order tensors are transformed into Cartesian components by the tensorial transformation law. 


\section{Appendix C The isochoric contributions of active sec-}

\section{ond Piola-Kirchhoff stress tensor $\boldsymbol{S}_{\text {act,iso }}$ and}

\section{fourth-order elasticity tensor $\mathbb{C}_{\text {act,iso }}$}

This Appendix gives explicit expressions for the isochoric contributions of the active second

Piola-Kirchhoff stress tensor $\boldsymbol{S}_{\text {act,iso }}$ and fourth-order elasticity tensor $\mathbb{C}_{\text {act,iso as stated in }}$

Section 3.3.

The isochoric contribution of the active second Piola-Kirchhoff stress tensor $\boldsymbol{S}_{\text {act,iso }}$ is given by

$$
\begin{aligned}
\boldsymbol{S}_{\text {act,iso }} & =2 \frac{\partial \Psi_{\mathrm{act}, \text { iso }}\left(\bar{\lambda}_{\mathrm{m}}\right)}{\partial \boldsymbol{C}}=J^{-2 / 3} \mathbb{P}: 2 \frac{\partial \Psi_{\mathrm{act}, \text { iso }}\left(\bar{\lambda}_{\mathrm{m}}\right)}{\partial \overline{\boldsymbol{C}}} \\
& =J^{-2 / 3} \mathbb{P}: \overline{\boldsymbol{S}}_{\mathrm{act}} .
\end{aligned}
$$

where the active fictitious second Piola-Kirchhoff stress tensor $\overline{\boldsymbol{S}}_{\text {act }}$ is defined as

$$
\begin{aligned}
\overline{\boldsymbol{S}}_{\mathrm{act}} & \equiv 2 \frac{\partial \Psi_{\mathrm{act}, \text { iso }}\left(\bar{\lambda}_{\mathrm{m}}\right)}{\partial \overline{\boldsymbol{C}}}=2 \frac{d \Psi_{\mathrm{act}, \text { iso }}\left(\bar{\lambda}_{\mathrm{m}}\right)}{d \bar{\lambda}_{\mathrm{m}}} \frac{\partial \bar{\lambda}_{\mathrm{m}}}{\partial \overline{\boldsymbol{C}}} \\
& =\frac{A}{\bar{\lambda}_{\mathrm{m}}}\left\{1-\left(\frac{\lambda_{\max }-\bar{\lambda}_{\mathrm{m}}}{\lambda_{\max }-\lambda_{0}}\right)^{2}\right\} \boldsymbol{m}_{0} \otimes \boldsymbol{m}_{0}
\end{aligned}
$$


Here we have used the following relationship:

$$
\frac{\partial \bar{\lambda}_{\mathrm{m}}}{\partial \overline{\boldsymbol{C}}}=\frac{1}{2 \bar{\lambda}_{\mathrm{m}}} \boldsymbol{m}_{0} \otimes \boldsymbol{m}_{0}
$$

The isochoric contribution of the active fourth-order elasticity tensor $\mathbb{C}_{\text {act,iso }}$ is given by

$$
\begin{aligned}
\mathbb{C}_{\mathrm{act}, \text { iso }}= & 2 \frac{\partial \boldsymbol{S}_{\mathrm{act}, \text { iso }}}{\partial \boldsymbol{C}}=4 \frac{\partial^{2} \Psi_{\mathrm{act}, \text { iso }}\left(\bar{\lambda}_{\mathrm{m}}\right)}{\partial \boldsymbol{C} \partial \boldsymbol{C}} \\
= & \mathbb{P}: 2 J^{-4 / 3} \frac{\partial \overline{\boldsymbol{S}}_{\mathrm{act}}}{\partial \overline{\boldsymbol{C}}}: \mathbb{P}^{\mathrm{T}}+\frac{2}{3}\left(J^{-2 / 3} \overline{\boldsymbol{S}}_{\mathrm{act}}: \boldsymbol{C}\right) \tilde{\mathbb{P}} \\
& -\frac{2}{3}\left(\boldsymbol{C}^{-1} \otimes \boldsymbol{S}_{\mathrm{act}, \text { iso }}+\boldsymbol{S}_{\mathrm{act}, \text { iso }} \otimes \boldsymbol{C}^{-1}\right) .
\end{aligned}
$$

Here we have used the following notation:

$$
\begin{aligned}
\frac{\partial \overline{\boldsymbol{S}}_{\mathrm{act}}}{\partial \overline{\boldsymbol{C}}}=\frac{A}{2}\left(-\frac{1}{\left(\bar{\lambda}_{\mathrm{m}}\right)^{3}}+\frac{\left(\lambda_{\max }-\bar{\lambda}_{\mathrm{m}}\right)^{2}}{\left(\bar{\lambda}_{\mathrm{m}}\right)^{3}\left(\lambda_{\max }-\lambda_{0}\right)^{2}}\right. & \\
& \left.\quad+\frac{2\left(\lambda_{\max }-\bar{\lambda}_{\mathrm{m}}\right)}{\left(\bar{\lambda}_{\mathrm{m}}\right)^{2}\left(\lambda_{\max }-\lambda_{0}\right)^{2}}\right)
\end{aligned}
$$

We remark that explicit expressions for the active fourth-order elasticity tensor have not been previously reported in the literature. 


\section{Appendix D Calculation of $\partial \Psi_{\text {pas }} / \partial \lambda_{\theta}, \lambda_{\theta} \partial \Psi_{\text {act }} / \partial \lambda_{\theta}$, and}

$$
\partial \Psi_{\mathrm{pas}} / \partial \lambda_{r}
$$

To obtain the analytical radius-pressure relationship stated in Section 4.1, we substitute the passive and active strain-energy function into Eq. (28). The calculation of $\partial \Psi_{\text {pas }} / \partial \lambda_{\theta}$, $\lambda_{\theta} \partial \Psi_{\text {act }} / \partial \lambda_{\theta}$, and $\partial \Psi_{\text {pas }} / \partial \lambda_{r}$ in Eq. (28) are presented in this Appendix.

First, we calculate $\partial \Psi_{\text {pas }} / \partial \lambda_{\theta}$ and $\partial \Psi_{\text {pas }} / \partial \lambda_{r}$ in Eq. (28). The first invariant of the right Cauchy-Green tensor $\boldsymbol{C}$ and the fourth and sixth pseudo-invariants of $\boldsymbol{C}, \boldsymbol{a}_{0} \otimes \boldsymbol{a}_{0}$, and $\boldsymbol{g}_{0} \otimes \boldsymbol{g}_{0}$ are respectively given by

$$
\begin{gathered}
I_{1}(r)=\lambda_{r}(r)^{2}+\lambda_{\theta}(r)^{2}+\lambda_{z}^{2}, \\
I_{4}(r)=\lambda_{\theta}(r)^{2} \cos ^{2} \eta_{j}+\lambda_{z}^{2} \sin ^{2} \eta_{j}, \\
I_{6}(r)=\lambda_{\theta}(r)^{2} \cos ^{2} \eta_{j}+\lambda_{z}^{2} \sin ^{2} \eta_{j} .
\end{gathered}
$$

The coupled form of the strain-energy function in the Holzapfel constitutive model is

$$
\Psi_{\mathrm{pas}}\left(\boldsymbol{C}, \boldsymbol{a}_{0} \otimes \boldsymbol{a}_{0}, \boldsymbol{g}_{0} \otimes \boldsymbol{g}_{0}\right)=c_{e}\left(I_{1}-3\right)+\frac{c_{1}}{2 c_{2}} \sum_{i=4,6}\left[\exp \left\{c_{2}\left(I_{i}-1\right)^{2}\right\}-1\right]
$$


Using Eqs. (D.1), (D.2), (D.3), and (D.4), we obtain

$$
\begin{aligned}
\frac{\partial \Psi_{\mathrm{pas}}}{\partial \lambda_{r}} & =\frac{\partial \Psi_{\mathrm{pas}}}{\partial I_{1}} \frac{\partial I_{1}}{\partial \lambda_{r}}+\frac{\partial \Psi_{\mathrm{pas}}}{\partial I_{4}} \frac{\partial I_{4}}{\partial \lambda_{r}}+\frac{\partial \Psi_{\mathrm{pas}}}{\partial I_{6}} \frac{\partial I_{6}}{\partial \lambda_{r}} \\
& =2 c_{e} \lambda_{r}(r)
\end{aligned}
$$

$$
\begin{aligned}
\frac{\partial \Psi_{\mathrm{pas}}}{\partial \lambda_{\theta}}= & \frac{\partial \Psi_{\mathrm{pas}}}{\partial I_{1}} \frac{\partial I_{1}}{\partial \lambda_{\theta}}+\frac{\partial \Psi_{\mathrm{pas}}}{\partial I_{4}} \frac{\partial I_{4}}{\partial \lambda_{\theta}}+\frac{\partial \Psi_{\mathrm{pas}}}{\partial I_{6}} \frac{\partial I_{6}}{\partial \lambda_{\theta}} \\
= & 2 c_{e} \lambda_{\theta}(r)+2 c_{1}\left\{I_{4}(r)-1\right\} \lambda_{\theta}(r) \cos ^{2} \eta_{j} \exp \left[c_{2}\left\{I_{4}(r)-1\right\}^{2}\right] \\
& +2 c_{1}\left\{I_{6}(r)-1\right\} \lambda_{\theta}(r) \cos ^{2} \eta_{j} \exp \left[c_{2}\left\{I_{6}(r)-1\right\}^{2}\right]
\end{aligned}
$$

where we have used the relationships

$$
\begin{gathered}
\frac{\partial I_{1}}{\partial \lambda_{r}}=2 \lambda_{r}(r), \quad \frac{\partial I_{1}}{\partial \lambda_{\theta}}=2 \lambda_{\theta}(r), \\
\frac{\partial I_{4}}{\partial \lambda_{r}}=\frac{\partial I_{6}}{\partial \lambda_{r}}=0, \quad \frac{\partial I_{4}}{\partial \lambda_{\theta}}=\frac{\partial I_{6}}{\partial \lambda_{\theta}}=2 \lambda_{\theta}(r) \cos ^{2} \eta_{j} .
\end{gathered}
$$

Next, we calculate of $\lambda_{\theta} \partial \Psi_{\text {act }} / \partial \lambda_{\theta}=\sigma_{\text {act }}^{\theta \theta}$ in Eq. (28). The coupled form of the strainenergy function in the active stress model is

$$
\Psi_{\mathrm{act}}\left(\lambda_{\mathrm{m}}\right)=A\left\{\lambda_{\mathrm{m}}+\frac{1}{3} \frac{\left(\lambda_{\max }-\lambda_{\mathrm{m}}\right)^{3}}{\left(\lambda_{\max }-\lambda_{0}\right)^{2}}\right\}
$$


The active second Piola-Kirchhoff stress tensor is given by

$$
\begin{aligned}
\boldsymbol{S}_{\mathrm{act}} & =2 \frac{\partial \Psi_{\mathrm{act}}\left(\lambda_{\mathrm{m}}\right)}{\partial \boldsymbol{C}}=2 \frac{d \Psi_{\mathrm{act}}\left(\lambda_{\mathrm{m}}\right)}{d \lambda_{\mathrm{m}}} \frac{\partial \lambda_{\mathrm{m}}}{\partial \boldsymbol{C}} \\
& =\frac{A}{\lambda_{\mathrm{m}}}\left\{1-\left(\frac{\lambda_{\max }-\lambda_{\mathrm{m}}}{\lambda_{\max }-\lambda_{0}}\right)^{2}\right\} \boldsymbol{m}_{0} \otimes \boldsymbol{m}_{0}
\end{aligned}
$$

where we have used the relationship

$$
\frac{\partial \lambda_{\mathrm{m}}}{\partial \boldsymbol{C}}=\frac{1}{2 \lambda_{\mathrm{m}}} \boldsymbol{m}_{0} \otimes \boldsymbol{m}_{0}
$$

The active Cauchy stress tensor $\boldsymbol{\sigma}_{\text {act }}$ is the push-forward operation of $\boldsymbol{S}_{\text {act }}$ by the deformation gradient tensor $\boldsymbol{F}$, i.e., $J \boldsymbol{\sigma}=\boldsymbol{F} \boldsymbol{S F}^{\mathrm{T}}$ (where $J=1$ in the incompressible condition). It is given by

$$
\begin{aligned}
\boldsymbol{\sigma}_{\mathrm{act}} & =\frac{A}{\lambda_{\mathrm{m}}}\left\{1-\left(\frac{\lambda_{\max }-\lambda_{\mathrm{m}}}{\lambda_{\max }-\lambda_{0}}\right)^{2}\right\} \boldsymbol{F}\left(\boldsymbol{m}_{0} \otimes \boldsymbol{m}_{0}\right) \boldsymbol{F}^{\mathrm{T}} \\
& =A \lambda_{\mathrm{m}}\left\{1-\left(\frac{\lambda_{\max }-\lambda_{\mathrm{m}}}{\lambda_{\max }-\lambda_{0}}\right)^{2}\right\} \boldsymbol{m} \otimes \boldsymbol{m} .
\end{aligned}
$$

Because smooth muscle cells are solely aligned in the circumferential direction and the boundary loadings are symmetric, we set $\lambda_{\mathrm{m}}=\lambda_{\theta}$ to obtain

$$
\sigma_{\mathrm{act}}^{\theta \theta}=A \lambda_{\theta}\left\{1-\left(\frac{\lambda_{\max }-\lambda_{\theta}}{\lambda_{\max }-\lambda_{0}}\right)^{2}\right\} .
$$




\section{References}

Alastrué V, Peña E, et al. 2007. Assessing the use of the“ opening angle method” to enforce residual stresses in patient-specific arteries. Ann. Biomed. Eng. 35(10):1821-1837.

Brink U and Stein E. 1996. On some mixed finite element methods for incompressible and nearly incompressible finite elasticity. Comput. Mech. 19(1):105-119.

Bustamante R and Holzapfel GA. 2010. Methods to compute 3D residual stress distributions in hyperelastic tubes with application to arterial walls. Intern. J. Eng. Sci. 48(11):10661082.

Chang T, Saleeb A, et al. 1991. Large strain analysis of rubber-like materials based on a perturbed Lagrangian variational principle. Comput. Mech. 8(4):221-233.

Chapelle D and Bathe K. 1993. The inf-sup test. Comput. Struct. 47(4-5):537-545.

Fung YC, Fronek K, et al. 1979. Pseudoelasticity of arteries and the choice of its mathematical expression. Am. J. Physiol. 237(5):H620-H631.

Fung YC and Liu SQ. 1989. Change of residual strains in arteries due to hypertrophy caused by aortic constriction. Circ. Res. 65(5):1340-1349.

Holzapfel GA. 2000. Nonlinear solid mechanics: A continuum approach for engineering. John Wiley \& Sons, LTD. 
Holzapfel GA, Gasser TC, et al. 2000. A new constitutive framework for arterial wall mechanics and a comparative study of material models. J. Elast. 61(1):1-48.

Holzapfel GA, Gasser TC, et al. 2002. A structural model for the viscoelastic behavior of arterial walls: continuum formulation and finite element analysis. Eur. J. Mech. -A/Solid. 21(3):441-463.

Holzapfel GA, Gasser TC, et al. 2004. Comparison of a multi-layer structural model for arterial walls with a fung-type model, and issues of material stability. J. Biomech. Eng. 126(2):264-275.

Humphrey JD and Na S. 2002. Elastodynamics and arterial wall stress. Ann. Biomed. Eng. $30(4): 509-523$.

Humphrey JD. 2003. Review Paper: Continuum biomechanics of soft biological tissues. Proceedings of the Royal Society of London. Series A Math., Physical. Eng. Sci. 459(2029):3-46.

Itskov M, Ehret AE, et al. 2006. A polyconvex anisotropic strain-energy function for soft collagenous tissues. Biomech. Model. Mechanobiol. 5(1):17-26.

Matsumoto T, Tsuchida M, et al. 1996. Change in intramural strain distribution in rat aorta due to smooth muscle contraction and relaxation. Am. J. Physiol. Heart. Circ. Physiol. 271(4):H1711-H1716. 
Murtada SI, Kroon M, et al. 2010. Modeling the dispersion effects of contractile fibers in smooth muscles J. Mech. Phys. Solids. 58(12):2065-2082.

Rachev A and Hayashi K. 1999. Theoretical study of the effects of vascular smooth muscle contraction on strain and stress distributions in arteries. Ann. Biomed. Eng. 27(4):459-468.

Rüter M and Stein E. 2000. Analysis, finite element computation and error estimation in transversely isotropic nearly incompressible finite elasticity. Comput. Methods. Appl. Mech. Eng. 190(5):519-541.

Schmitz A and Böl M. 2011. On a phenomenological model for active smooth muscle contraction. J. Biomech. 44(11):2090-2095.

Schöder J and Neff P. 2003. Invariant formulation of hyperelastic transverse isotropy based on polyconvex free energy functions. Intern. J. Solid. Struct. 40(2):401-445.

Schröder J, Neff P, et al. 2005. A variational approach for materially stable anisotropic hyperelasticity. Intern. J. Solid. Struct. 42(15):4352-4371.

Silvester DJ and Kechkar N. 1990. Stabilised bilinear-constant velocity-pressure finite elements for the conjugate gradient solution of the Stokes problem. Comput. Methods. Appl. Mech. Eng. 79(1):71-86.

Stålhand J, Klarbring A, et al. 2011. A mechanochemical 3D continuum model for smooth muscle contraction under finite strains. J. TheoBiol. 268(1):120-130. 
Sussman T and Bathe KJ. 1987. A finite element formulation for nonlinear incompressible elastic and inelastic analysis. Comput. Struct. 26(1-2):357-409.

Takamizawa K, Hayashi K, et al. 1992. Isometric biaxial tension of smooth muscle in isolated cylindrical segments of rabbit arteries. Am. J. Physio.l Heart. Circ. Physiol. 263(1):H30H34.

Vaishnav RN and Vossoughi J. 1987. Residual stress and strain in aortic segments. J. Biomech. 20(3):235-239.

Van Dyke TJ and Hoger A. 2002. A new method for predicting the opening angle for soft tissues. J. Biomech. Eng. 124(4):347-354.

Vincent C and Boyer R. 1992. A preconditioned conjugate gradient Uzawa- type method for the solution of the stokes problem by mixed Q1-P0 stabilized finite elements. Intern. J. Numer. Methods. Fluid. 14(3):289-298.

Wang I, Politi AZ, et al. 2008. A mathematical model of airway and pulmonary arteriole smooth muscle. Biophys. J. 94(6):2053-2064.

Weiss JA, Maker BN, et al. 1996. Finite element implementation of incompressible, transversely isotropic hyperelasticity. Comput. Methods. Biomech. Eng. 14(9):803-817.

Weiss JA, Gardiner JC, et al. 2005. Three-dimensional finite element modeling of ligaments: Technical aspects. Med. Eng. Phys. 27(10):845-61. 
Zulliger MA, Rachev A, et al. 2004. A constitutive formulation of arterial mechanics including vascular smooth muscle tone. Am. J. Physiol. Heart. Circ. Physiol. 287(3):H1335-H1343. 


\section{Table 1}

Material constants used in the passive constitutive law Eq. (10) taken from previous studies on rabbit carotid artery (Holzapfel et al., 2004).

\begin{tabular}{ccccc}
\hline Layer & $c_{e}[\mathrm{kPa}]$ & $c_{1}[\mathrm{kPa}]$ & $c_{2}$ & $\eta[\mathrm{deg}]$ \\
\hline \hline Inner & 3.380 & 5.399 & 0.3579 & 20 \\
Outer & 0.3831 & 0.8255 & 1.030 & 65 \\
\hline
\end{tabular}




\section{Table 2}

Material constants used in the active constitutive law Eq. (18) taken from previous studies

(Rachev et al., 1999).

\begin{tabular}{cccc}
\hline Muscle tone & $A[\mathrm{kPa}]$ & $\lambda_{\max }$ & $\lambda_{0}$ \\
\hline \hline Basal tone & 50 & 1.4 & 0.65 \\
\hline
\end{tabular}




\section{Table 3}

Geometric arterial segment data of rabbit carotid artery (Holzapfel et al., 2000, 2004).

State Opening angle $[\mathrm{deg}] \quad$ Inner radius $[\mathrm{mm}] \quad$ Outer radius $[\mathrm{mm}]$

\begin{tabular}{lccc}
\hline \hline Stress free & 160 & 1.43 & 1.82 \\
Load free & - & 0.71 & 1.10 \\
\hline
\end{tabular}




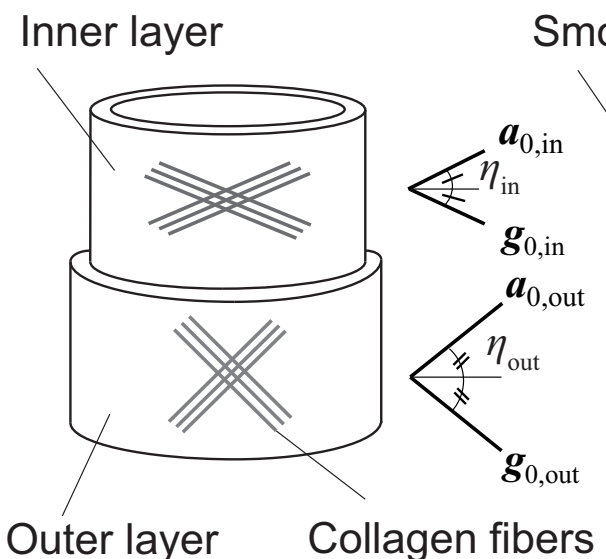

Smooth muscle cell

Figure 1: Computational model of arterial wall. The arterial wall comprises two layers embedding active stress generators, i.e., smooth muscle cells. Each layer is composed of a non-collagenous matrix and two families of collagen fibers, whose reference directions are represented by unit vectors $\boldsymbol{a}_{0}$ and $\boldsymbol{g}_{0}$. Smooth muscle cells, whose reference directions are represented by the unit vector $\boldsymbol{m}_{0}$, mainly align in the circumferential direction and predominantly generate circumferential active stress. 

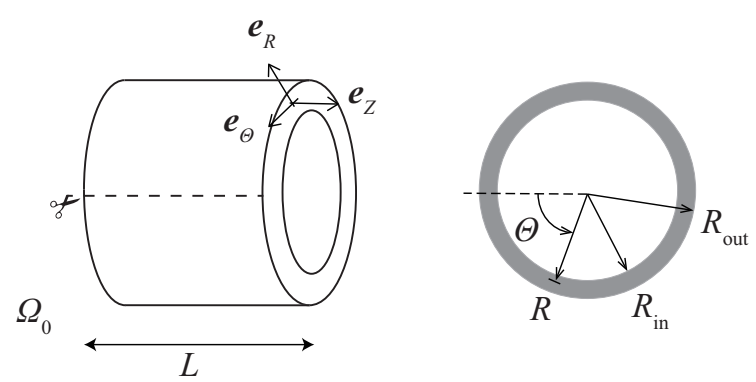

(a) Arterial segment excised from the body. Reference configuration $\Omega_{0}$. Closed load-free state. The set $\left\{\boldsymbol{e}_{r}, \boldsymbol{e}_{\theta}, \boldsymbol{e}_{z}\right\}$ is the local covariant basis of the cylindrical coordinate system. The contravariant components of a material point vector are denoted as $(R, \Theta, Z)$.

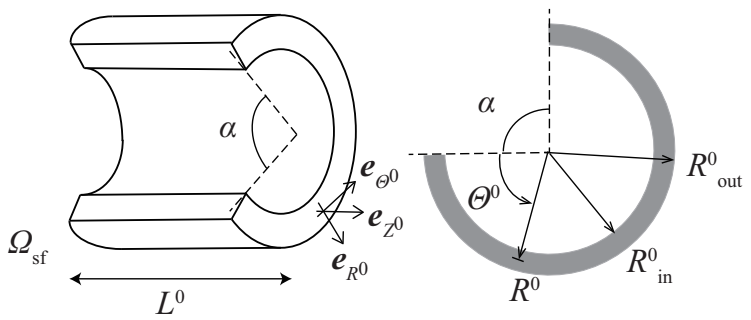

(b) Radial cut. Stress-free configuration $\Omega_{\mathrm{sf}}$. Opened up stress-free state. The set $\left\{\boldsymbol{e}_{R^{0}}, \boldsymbol{e}_{\Theta^{0}}, \boldsymbol{e}_{Z^{0}}\right\}$ is the local covariant basis of the cylindrical coordinate system. The contravariant components of a stress-free material point vector are denoted as $\left(R^{0}, \Theta^{0}, Z^{0}\right)$.

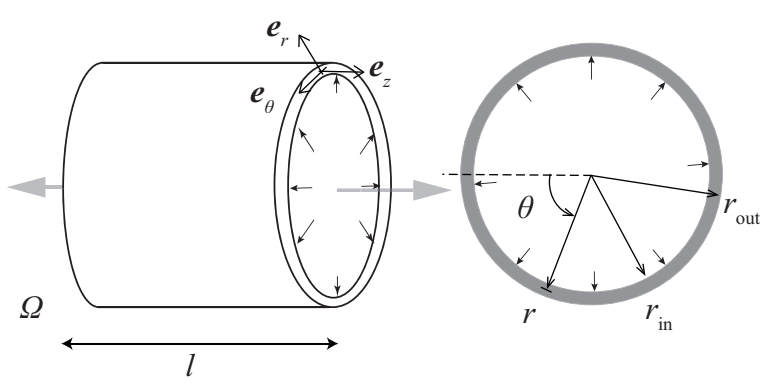

(c) Application of external loads. Current configuration $\Omega$. Loaded deformed state. The set $\left\{\boldsymbol{e}_{r}, \boldsymbol{e}_{\theta}, \boldsymbol{e}_{z}\right\}$ is the local covariant basis of the cylindrical coordinate system. The contravariant components of a spatial point vector are denoted as $(r, \theta, z)$.

Figure 2: Schematic of the opening angle method for assessing the residual deformation gradient $\boldsymbol{F}_{\text {res }}$ in cylindrical geometry. The arterial segment is assumed to be axially uniform; therefore the opening angle $\alpha$ is independent of axial position $Z^{0}$. 


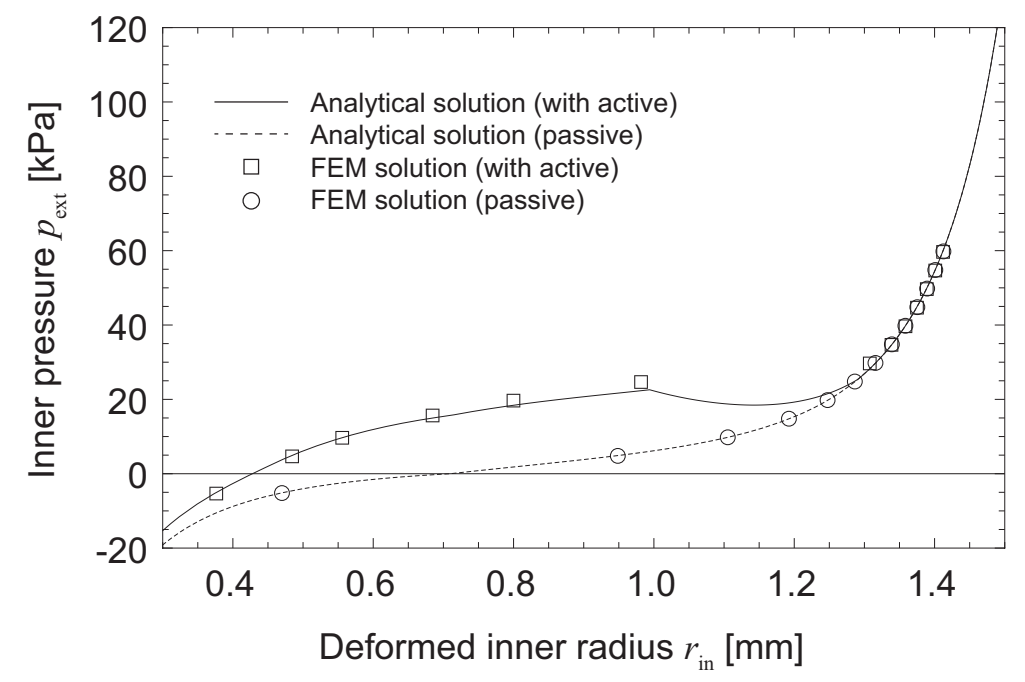

Figure 3: Deformed inner radius vs. external pressure loads (inner pressure). Solid and dashed lines indicate the exact solution of the Holzapfel constitutive model plus the active stress model $(A=50 \mathrm{kPa})$ and the (passive) Holzapfel constitutive model, respectively. The symbols $\square$ and $\circ$ indicate corresponding finite element solutions. 


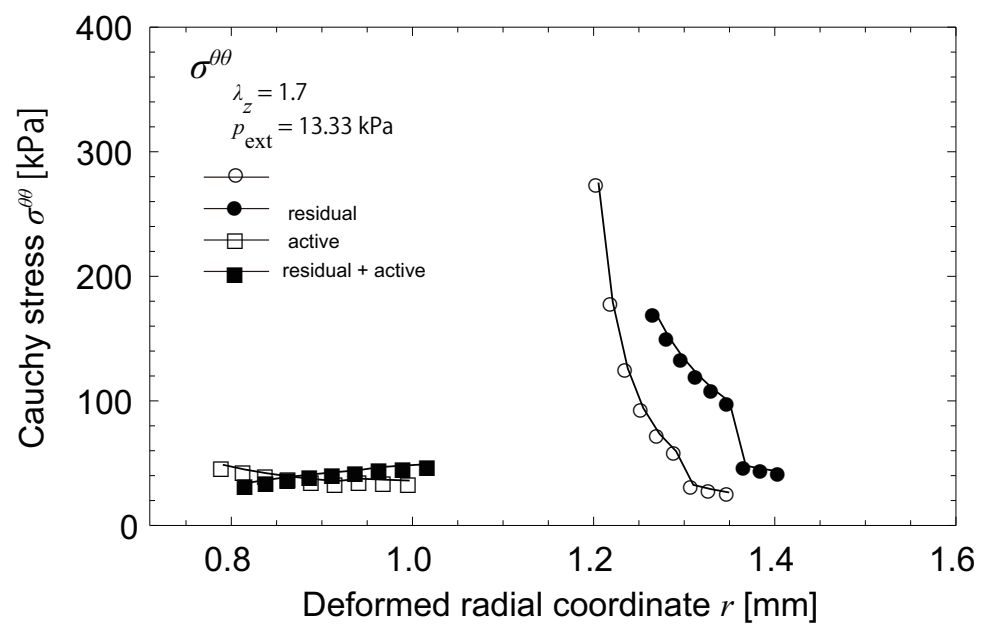

(a) Circumferential direction.

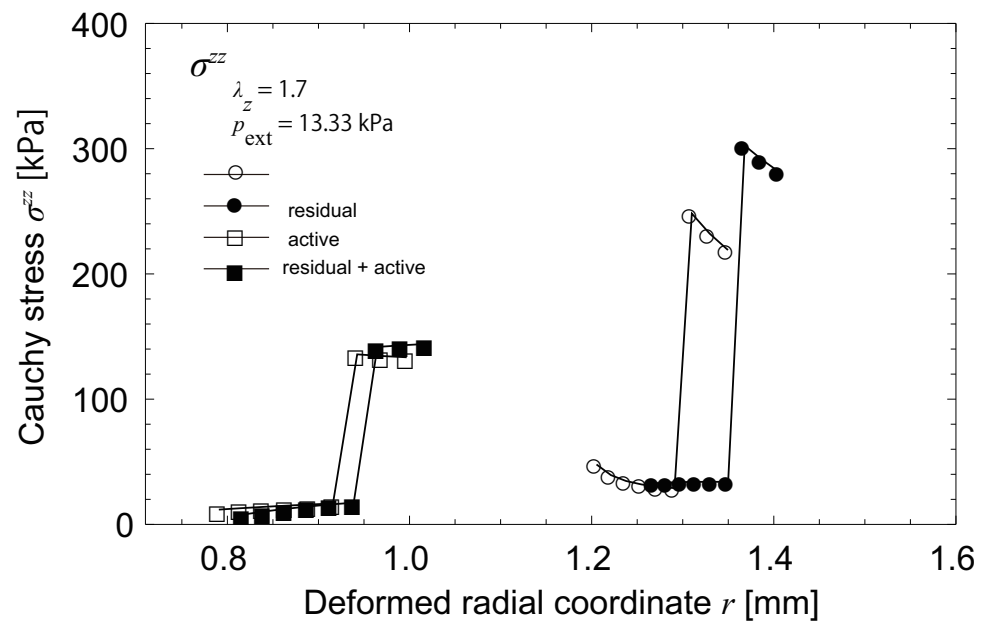

(b) Axial direction.

Figure 4: Comparison of Cauchy stress distributions in the presence/absence of residual and/or active stresses at normal external pressure load $p_{\text {ext }}=13.33 \mathrm{kPa}$.

Case 1(०): No residual or active stresses.

Case 2(•): Residual stresses present; active stresses absent.

Case 3( $\square$ ): Residual stresses absent; active stresses present.

Case 4(ם): Presence of residual and active stresses. 


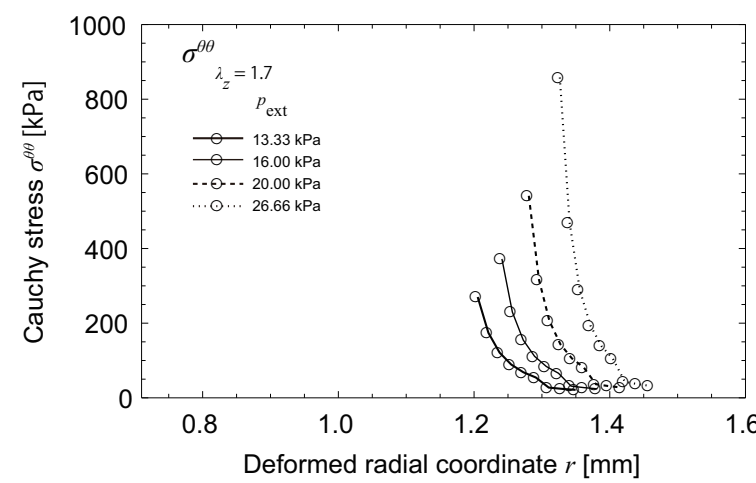

(a) Absence of residual and active stresses.

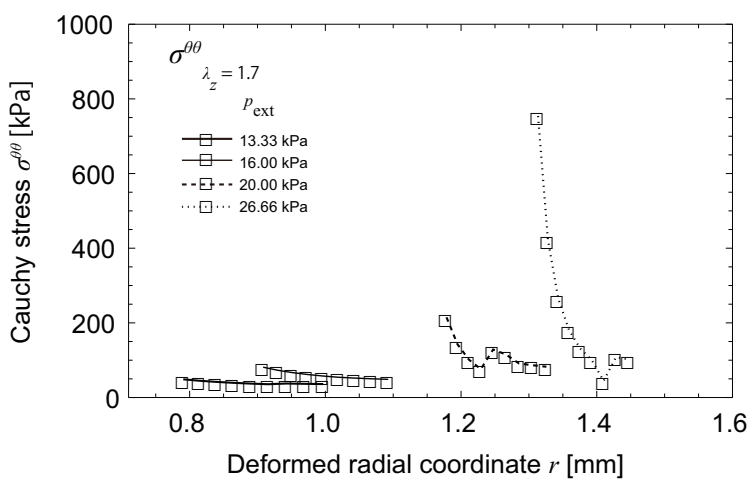

(c) Residual stresses absent; active stresses present.

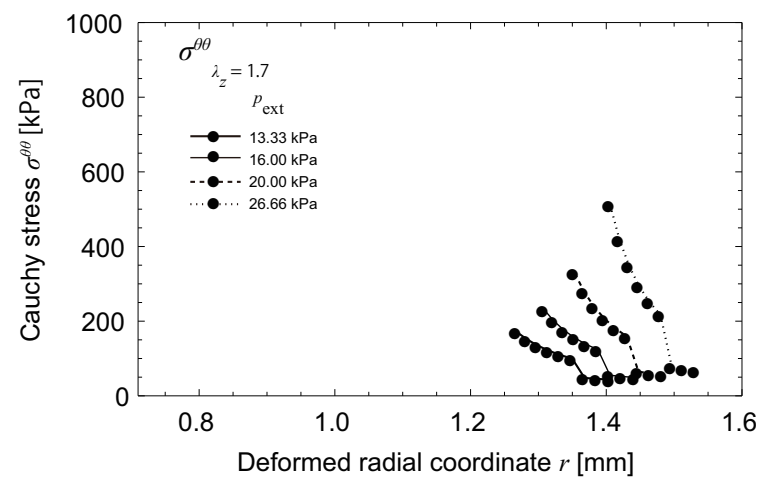

(b) Residual stresses present; active stresses absent.

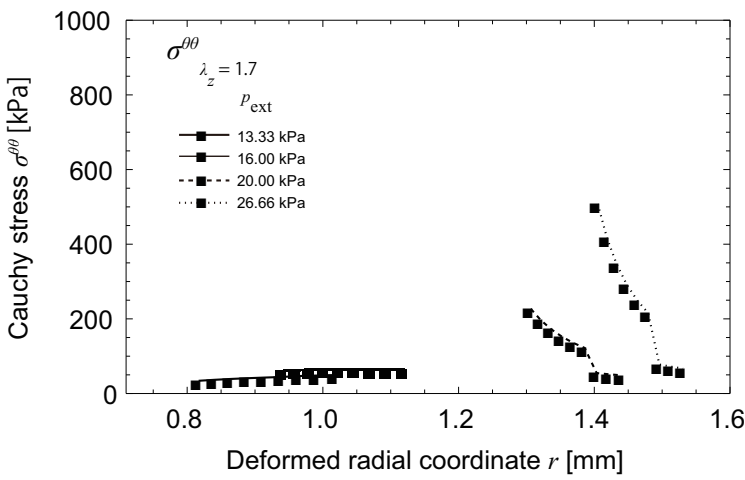

(d) Presence of residual and active stresses.

Figure 5: Comparison of circumferential Cauchy stress distributions in the presence/absence of residual and/or active stresses under various external pressure loads ranging from 13.33 to $26.66 \mathrm{kPa}$.
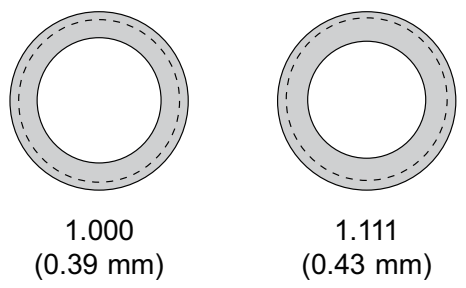

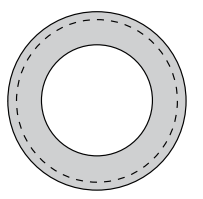

1.222

$(0.47 \mathrm{~mm})$

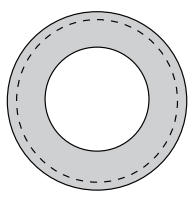

1.333

$(0.51 \mathrm{~mm})$

Figure 6: Arteries respond to the hypertensive state by increasing their wall thickness toward the inside of the cavity. The numbers beneath the artery cross-sections in this figure indicate the wall thickness resulting from hypertrophy. 


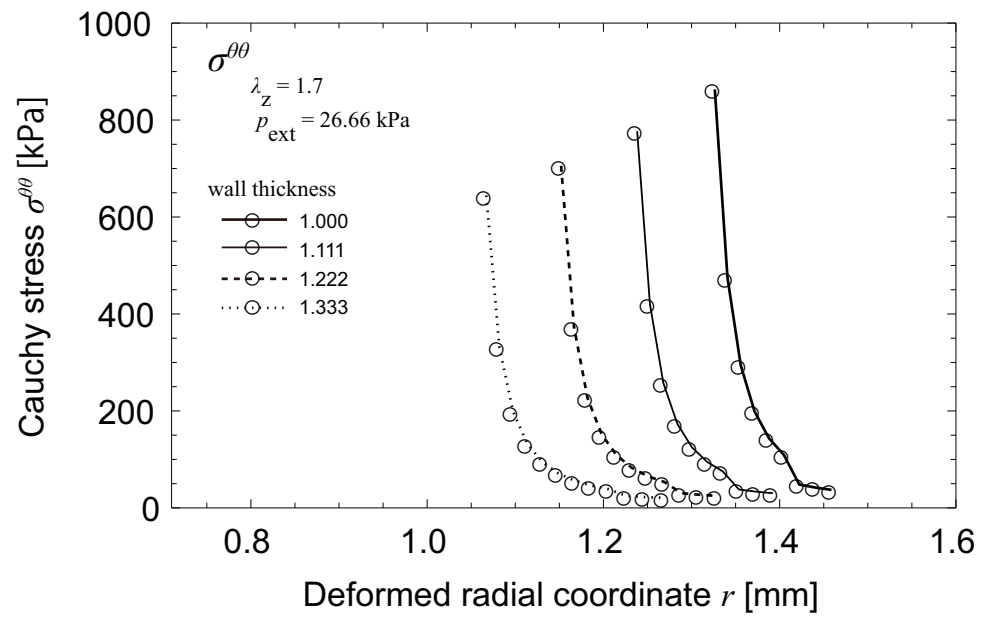

(a) Absence of residual and active stresses.

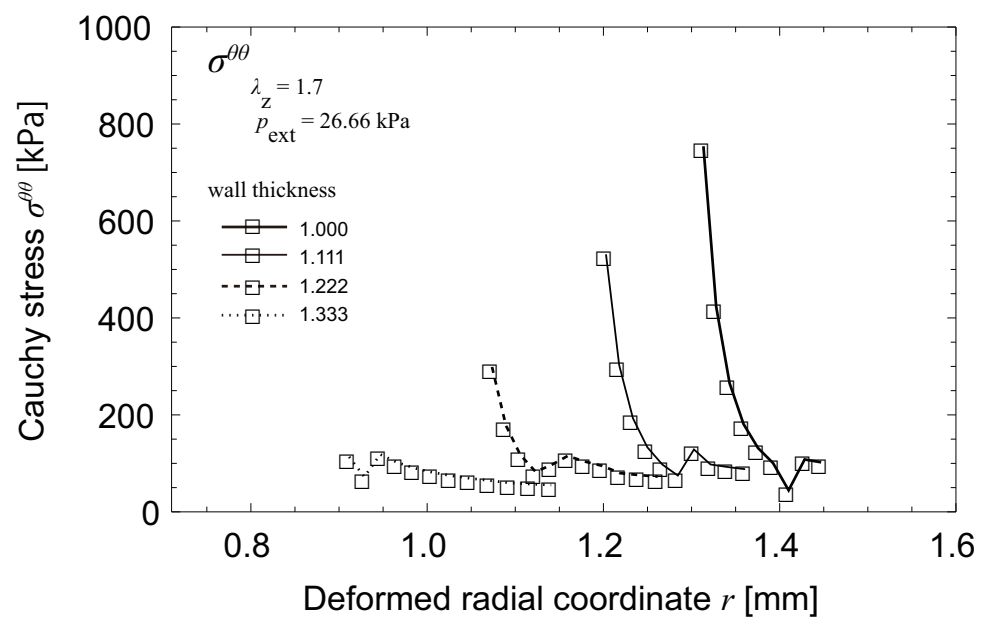

(b) Residual stresses absent; active stresses present.

Figure 7: Comparison of circumferential Cauchy stress distributions at severe hypertensive loading $\left(p_{\text {ext }}=26.66 \mathrm{kPa}\right)$ as functions of deviations in the arterial wall thickness. 